\title{
Numerical prediction of eddy structure in a shear-driven cavity
}

\author{
T. P. Chiang, W. H. Sheu
}

\begin{abstract}
We present in this paper a detailed numerical study of the vortical flow structure in a confined lid-driven cavity which is defined by a depth-to-width aspect ratio of $1: 1$ and a span-to-width aspect ratio of 3:1. In this study we have carefully examined the computed data that the useful to gain an in-depth knowledge of the complex interactions among secondary eddies, primary eddies, and spiraling spanwise motions. Chief of conclusions drawn from this study is to explain how the secondary eddies are intimately coupled with the primary recirculating flow. We also enlighten in this paper why spiraling vortices inside the upstream secondary eddy tend to destabilize the incompressible flow system and aid development of laminar instabilities.
\end{abstract}

\section{1}

\section{Introduction}

Over the last three decades, the so-called lid-driven cavity flow problem has received considerable attention mainly because of its geometric simplicity, physical abundance, and its close relevance to some fundamental engineering flows. Numerical investigation of this problem dates back to the pioneer work of Burgraff (1966). While some fundamental flow phenomena have become clear to us through two-dimensional solutions, many of the subtleties of third-dimensionality are yet to be learned. The recent progress in numerical analyses and computer hardware have made it possible to numerically analyze unsteady flow problems by solving their corresponding Navier-Stokes equations with a large number of grid points within a three-dimensional domain.

In a parallel development, a considerable number of experimental studies on this problem have been done since the early 1980s. Among them, Taylor-Görtler-like (TGL) vortices were experimentally observed by Koseff et al. (1983). Later in 1985, TGL vortices were numerically confirmed by Freitas et al. (1985). For a comprehensive literature review, see the research work of Street and his colleagues (Koseff and Street 1982; Koseff et al. 1983; Rhee

Communicated by T. Tezduyar, 18 February 1997

T. P. Chiang, W. H. Sheu

Department of Naval Architecture and Ocean Engineering,

National Taiwan University,

73 Chou-Shan Rd. Taipei, Taiwan, R. O. C. et al. 1984; Koseff and Street 1984; Prasad et al. 1988; Prasad and Koseff 1989; Freitas et al. 1985; Freitas and Street 1988; Perng and Street 1989; Zang et al. 1993).

We review here mainly the published work of the research group led by Street. Experimental investigation of cavity flows for Reynolds numbers between 1000 and 10000 started in the early 1980s. Koseff and Street (1984) observed corner vortices in the vicinity of the two vertical end walls and local TGL vortices over the span of the cavity with the values of SAR (Spanwise Aspect Ratio) ( $\equiv \mathrm{L}: \mathrm{B}=1,2,3$ ). In the case of $\mathrm{Re} \sim 3000$, eight pairs of TGL vortices were observed. On increasing Re up to 6000 , three more pairs (11 pairs) of TGL vortices became visible. For Reynolds numbers as high as $6000 \sim 8000$, unsteadiness no longer was regularly sustained and, thus, evolved into turbulence. Spiraling spanwise motion has been discussed mainly inside the downstream secondary eddy (DSE). To the best of the authors' knowledge, previous studies have never addressed the upstream secondary eddy (USE). In 1988-1989, Reynolds numbers falling into the range of $3200 \sim 10000$ were considered by Prasad et al. (1988) and Prasad and Koseff (1989). In their studies, different values of SAR $=1: 2,2: 3,5: 6$, and $1: 1$ were considered with particular emphasis on study of the influence of the reduced values of SAR on the increase of the viscous drag. More recently, Reynolds numbers classified as low to medium $(100 \sim 2000)$ were considered by Aidun et al. (1991). Their paper classified the flow in terms of Reynolds numbers.

In 1991, the GAMM-Committee sponsored a workshop dedicated to numerical simulation of a lid-driven cavity flow at $\mathrm{Re}=3200$ for $\mathrm{SAR}=3: 1$ (Deville et al. 1992) with the aim of ascertaining the number of TGL vortex pairs appearing in the transverse direction. Comparisons were also made among participants on the performance of the computer codes employed and on the CPU time per unit of physical time. It was surprising to find from the summary of this conference is that the conclusions drawn were quite different among the contributors. They differed not only on the existence of the flow symmetry, but also on the number of pairs of TGL vortices. In recognition of this, we feel that much work needs to be performed in the years ahead. To avoid physical complications and numerical difficulties, we consider here a Reynolds number that is much lower than 3200. For numerical simulations of cavity flow at Reynolds numbers other than 3200, the reader may refer to Deng et al. (1994).

We begin by introducing the equations of the fluid motion for the incompressible case described in Sect. 2. 
This is followed by a brief description of the underlying finite volume discretization method, the solution algorithm, and the multi-dimensional advective flux discretization scheme. For confident use of the developed computer code for the lid-driven cavity problem, validation against analytic data is presented in Sect. 3. In Sect. 4, prior to discussing in detail the complex flow evolution, we compare solutions with other numerically available liddriven cavity data. Attention is given to the interaction mechanism between secondary eddies and primary circulation. Plausible reasons leading to the formation of TGL vortices are also addressed. Closing remarks are presented in Sect. 5.

\section{2}

\section{Mathematical model and numerical method}

We consider in this paper the flow of an incompressible, viscous fluid. In the absence of an applied body force, a complete set of conservation equations is given by the following elliptic-parabolic equations for a given Reynolds number Re:

$$
\begin{aligned}
& \frac{\partial u_{i}}{\partial x_{i}}=0, \\
& \frac{\partial u_{i}}{\partial t}+\frac{\partial}{\partial x_{m}}\left(u_{m} u_{i}\right)=-\frac{\partial p}{\partial x_{i}}+\frac{1}{\operatorname{Re}} \frac{\partial^{2} u_{i}}{\partial x_{m} \partial x_{m}} .
\end{aligned}
$$

In order to be closed, Eqs. (1), (2) with an initial condition at $t=0$ must be supplemented by boundary values. In the literature, we find several sets of working variables to choose from. Of these, we prefer to employ the primitivevariable formulation for this class of flows. The rationale behind choosing this variable setting is that closure boundary and initial conditions are clearly defined (Ladyzhenskaya 1963). More importantly, these closure conditions are relatively easy to be implemented in the computer code.

When faced with incompressible Navier-Stokes

Eqs. (1-2), we encounter two well-known difficulties. The first of the two deals with the numerical instability in situations when advection predominates over diffusion. Prediction error due to oscillatory velocities and the socalled false diffusion error grossly pollute the flow physics over the entire domain. A simple remedy for such discretization errors is to apply the third-order QUICK (Leonard 1979) upwind scheme, formulated on the nonuniform basis, to the nonlinear advective fluxes. It must be borne in mind that discretization errors stemming from the use of a one-to-one curvilinear coordinate transformation are considerable and cause deterioration of accuracy for problems involving highly stretched and distorted meshes (Sheu and Lee 1996). In attempting to explore in depth how the flow evolves, we conduct analysis in a cavity containing a simple set of rectangular grids.

When the velocity-pressure formulation is considered, one has a choice of applying a grid staggering (Patankar 1980) or a collocating (Abdallah 1987) strategy to storing these working variables. While use of both approaches to suppress node-to-node pressure oscillations has long been recognized in the literature, we abandon the collocating grid approach in favor of the first strategy. Regardless of programming complications, the staggered grid approach outweighs the nonstaggered grid in the sense that the latter approach lacks boundary pressure for an analysis involving a Poisson-type pressure correction equation. On the control surface of a finite volume cell, each primitive variable takes over a node to itself whereas the pressure node is surrounded by nodal velocities. This variable setting permits the use of the finite volume integration method to discretize each conservation equation under these circumstances.

The second difficulty encountered in simulating an incompressible flow problem is that it is essential to assure satisfaction of discrete divergence-free velocities. While use of a mixed formulation meets this constraint condition, we encounter a much larger discrete system. The need to avoid a much larger demand for computer storage has prompted researchers to consider segregated approaches. In the present paper, the solution to the finite volume discretization equations is obtained sequentially for all primitive variables using the underlying SIMPLE iterative algorithm (Patankar 1980).

\section{3}

\section{Validation study}

As a first step towards numerical investigation of the physical problem, we first justify our computer code. A validation study is often proceeded by an analytical assessment of the problem. To achieve this goal and, furthermore, estimate the spatial rate of convergence of the scheme employed, we consider the following transport equation for a scalar $\Phi$ in a simple domain of three dimensions $(-1 \leq x, y, z \leq 1)$. Of note is that $\partial \Phi / \partial t$ has been analytically specified:

$$
\frac{\partial \Phi}{\partial t}+\operatorname{Re} \frac{\partial}{\partial x_{j}}\left(u_{j} \Phi\right)=\frac{\partial^{2} \Phi}{\partial x_{j} \partial x_{j}}+S ; \quad(j=1,2,3) .
$$

Here, $u_{j}, \operatorname{Re}$, and $S$ denote the velocity components of the velocity vector, the Reynolds number, and the source per unit volume, respectively. The solution to Eq. (3) is sought in a domain with the following zero-divergence velocity field (Ethier and Steinman 1994):

$$
\begin{aligned}
u & =-a\left[\mathrm{e}^{a x} \sin (a y \pm \mathrm{d} z)+\mathrm{e}^{a z} \cos (a x \pm \mathrm{d} y)\right] \mathrm{e}^{-d^{2} t} \\
v & =-a\left[\mathrm{e}^{a y} \sin (a z \pm \mathrm{d} x)+\mathrm{e}^{a x} \cos (a y \pm \mathrm{d} z)\right] \mathrm{e}^{-d^{2} t} \\
w & =-a\left[\mathrm{e}^{a z} \sin (a x \pm \mathrm{d} y)+\mathrm{e}^{a y} \cos (a z \pm \mathrm{d} x)\right] \mathrm{e}^{-d^{2} t}
\end{aligned}
$$

where $a=\frac{d}{2}=\frac{\pi}{4}$. The analytic solution for $\Phi$, thus, takes the same form as $u$ given in (4) if the source term $S$ is prescribed as follows:

$S=-\operatorname{Re} \frac{\partial \mathrm{p}}{\partial \mathrm{x}}$

where

$$
\begin{aligned}
p= & -\frac{a^{2}}{2}\left[\mathrm{e}^{2 a x}+\mathrm{e}^{2 a y}+e^{2 a z}\right. \\
& +2 \sin (a x \pm \mathrm{d} y) \cos (a z \pm \mathrm{d} x) \mathrm{e}^{a(y+z)} \\
& +2 \sin (a y \pm \mathrm{d} z) \cos (a x \pm \mathrm{d} y) \mathrm{e}^{a(z+x)} \\
& \left.+2 \sin (a z \pm \mathrm{d} x) \cos (a y \pm \mathrm{d} z) \mathrm{e}^{a(x+y)}\right] \mathrm{e}^{-2 d^{2} t}
\end{aligned}
$$


As is usual, we assessed the employed QUICK-type upwind discretization scheme by examining the prediction nodal errors. Tests on various grids were conducted to assure that the solution converged. With grid spacings being continuously refined, we could compute the rate of convergence from the computed $L_{2}$-error norms. According to the errors computed at different grid spacings, namely $h=1,2 / 3,1 / 2,2 / 5,1 / 3,2 / 7$, the ratio of convergence as shown in Fig. 1 is, as expected, 3 in a least-square sense. The test case considered and the results obtained thus far confirm the applicability of the QUICK scheme to multidimensional analyses.

We now turn to examining whether or not linearization procedures and the zero-divergence constraint condition will cause the rate of convergence to deteriorate. To answer, this question, we solved a Navier-Stokes problem in the same domain as that considered in the previous benchmark test. For this study, this involves specifying a Dirichlet-type velocity and initial condition according to equations given in (4). The analytic pressure takes exactly the same form as that shown in Eq. (6). This is only true for the case with $\mathrm{Re}=1$ (Ethier and Steinman 1994). We carried out the computations under conditions of time spacing $\Delta t=1 / 160$ and the grid spacing considered in the previous benchmark test. From the finite volume solutions shown in Fig. 2, we are assured that the proposed scheme is also applicable to analysis of incompressible NavierStokes equations. The good agreement from two analytical tests, as demonstrated in Figs. 1-2, provides us with strong confidence to proceed with investigation of the time-history of the flow evolution, which is driven by a constant upper lid, in the rectangular cavity.

\section{4}

\section{Results and discussion}

\section{1}

\section{Problem description and solution validation}

The problem under investigation, as configured in Fig. 3, concerns the incompressible fluid flow inside a rectangular cavity, which is scaled by $\mathrm{L}: \mathrm{B}: \mathrm{D}=3: 1: 1$. The Reynolds

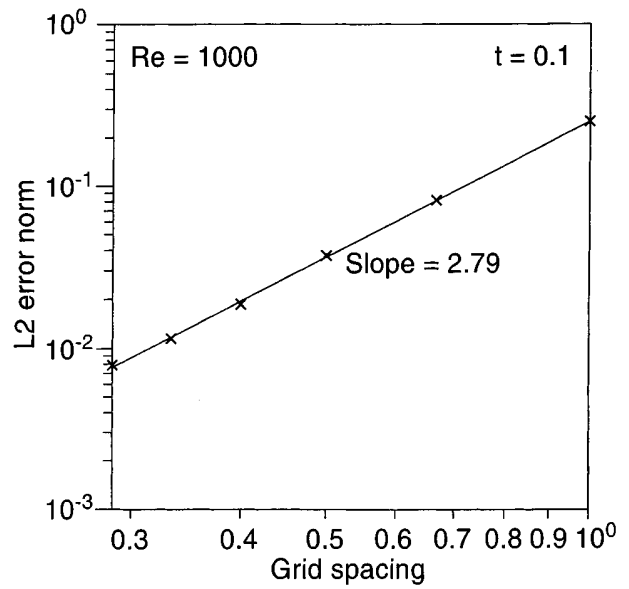

Fig. 1. The rate of the spatial convergence test for a threedimensional scalar equation

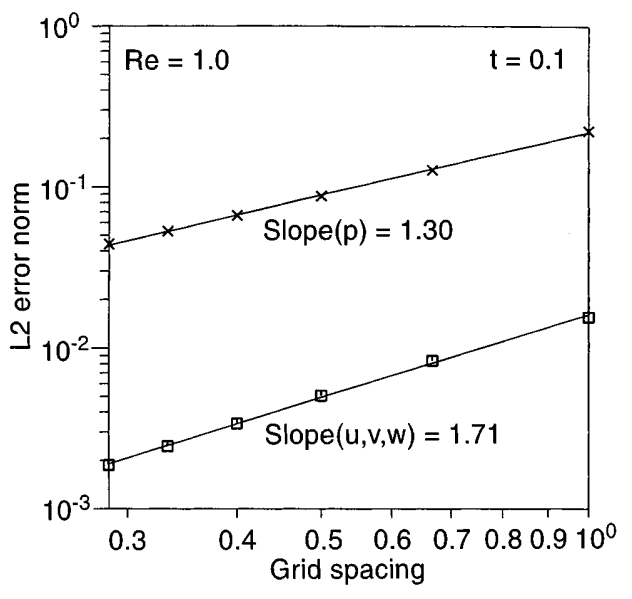

Fig. 2. The computed rates of convergence for velocities and pressure for the Navier-Stokes equations defined in a cubic

number chosen for this cavity is based on the lid speed, the width of the cavity, and the kinematic viscosity of the working fluid. According to Fig. 3, the cavity of present interest is subject to a sudden upper lid motion at time $t=0$.

Recalling the ambiguity regarding whether or not the flow symmetry is retained in the investigated rectangular cavity, we have conducted numerical analysis in the whole cavity, which is covered with non-uniform mesh with $34 \times 91 \times 34$ grid resolution, to avoid such uncertainty. Computational evidence reveals that Taylor-Görtler vortices emerge at a Reynolds number around 1300. This finding is consistent with that of Aidun, Triantafillopoulos, and Benson (1991). With a continuous increase of the Reynolds number, unsteady flow gradually evolves into turbulence at $\mathrm{Re}=6000$. In recognition of these findings, we address a Reynolds number whose value is not so high as to render a turbulent flow because obstacles regarding turbulence modeling remain on the road to a realistic simulation. In circumstances where the Reynolds number is much less than 1300, TGL vortices are not expected to appear, so lower Reynolds numbers are, thus, not considered here. In attempting to explore in depth the mechanism of laminar instability leading to the onset of TGL vortices, we mainly consider here a moderate Reynolds number, $\mathrm{Re}=1500$.

Prior to discussion of the computed solutions, we will describe tests on different spanwise ratios, namely $\mathrm{SAR}=1,3$, and Reynolds numbers, namely 400, 1000, 3200 , which were carried out to further show that our code is applicable to the lid-driven cavity problem. A comparison was made on the basis of mid-span velocity profiles along vertical as well as horizontal centerlines. According to the computed finite volume solutions and their convergence histories, as depicted in Figs. 4-6, for the cavities considered and the Reynolds numbers investigated, the agreement with other numerical solutions (Ku et al. 1987; Kato et al. 1990; Cortes and Miller 1994; Babu and Korpela 1994; Arnal et al. 1992; Kost et al. 1992) is close enough. In the remaining sections, we will focus mainly on some topics which have been little explored. 


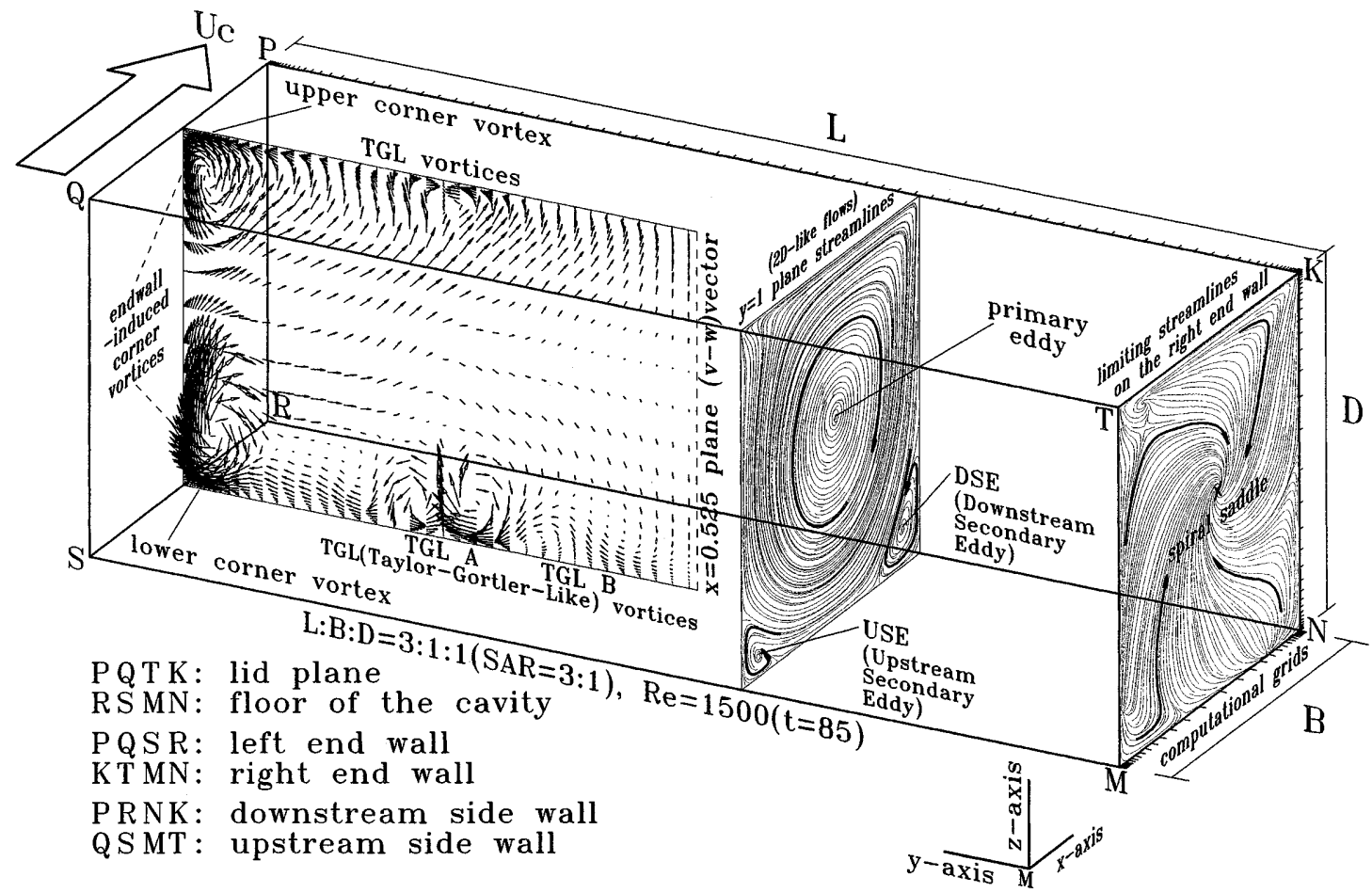

Fig. 3. An illustration of the flow structure in the investigated rectangular cavity
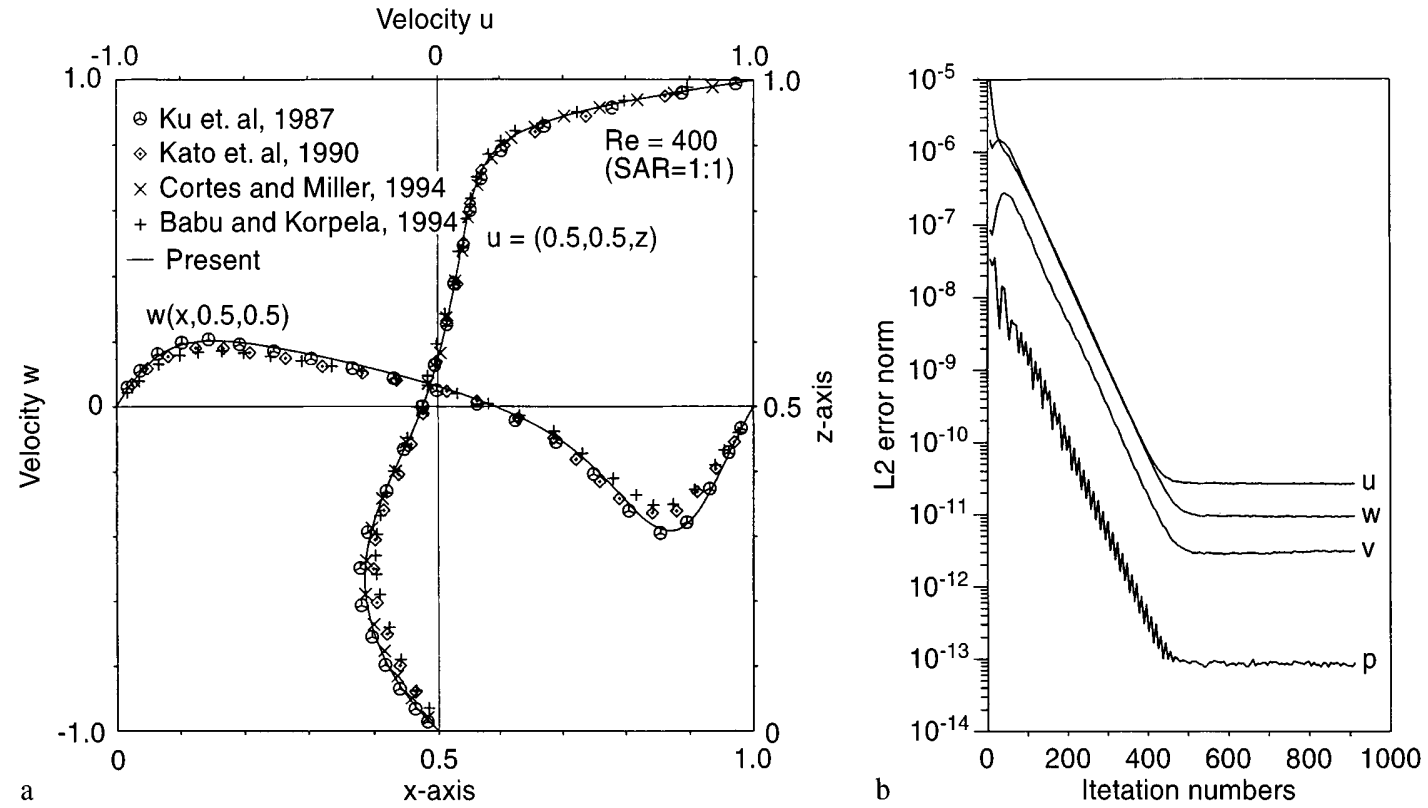

Fig. 4 a,b. Comparison study for $\mathrm{SAR}=1: 1$ and $\mathrm{Re}=400$. a mid-sectional velocities at the symmetry plane $y=0.5$; $\mathbf{b}$ error reduction for working variables

\section{2}

\section{Global flow structure}

To provide readers with a clear picture of the flow structure in the cavity of interest, we present in Fig. 3 a global view of the flow at $t=85$. In $y$ planes that are sufficiently distant from the two end walls, say at the $y=1$ spanwise plane, the flow pattern resembles that computed on a twodimensional basis which comprises a primary eddy and two much smaller secondary eddies, namely the down- stream secondary eddy (DSE) and the upstream secondary eddy (USE). The two-dimensional-like streamlines plotted in Fig. 3 differ from truly two-dimensional ones in that the streamlines are open (not close together), so they permit the presence of spanwise velocity component. No Moffatt vortex is found unless the Reynolds number approaches 3000. Due to the stagnant nature of the flow in regions fairly close to the two end walls, in the direction away from the two end walls there exists a negative pressure gradient 

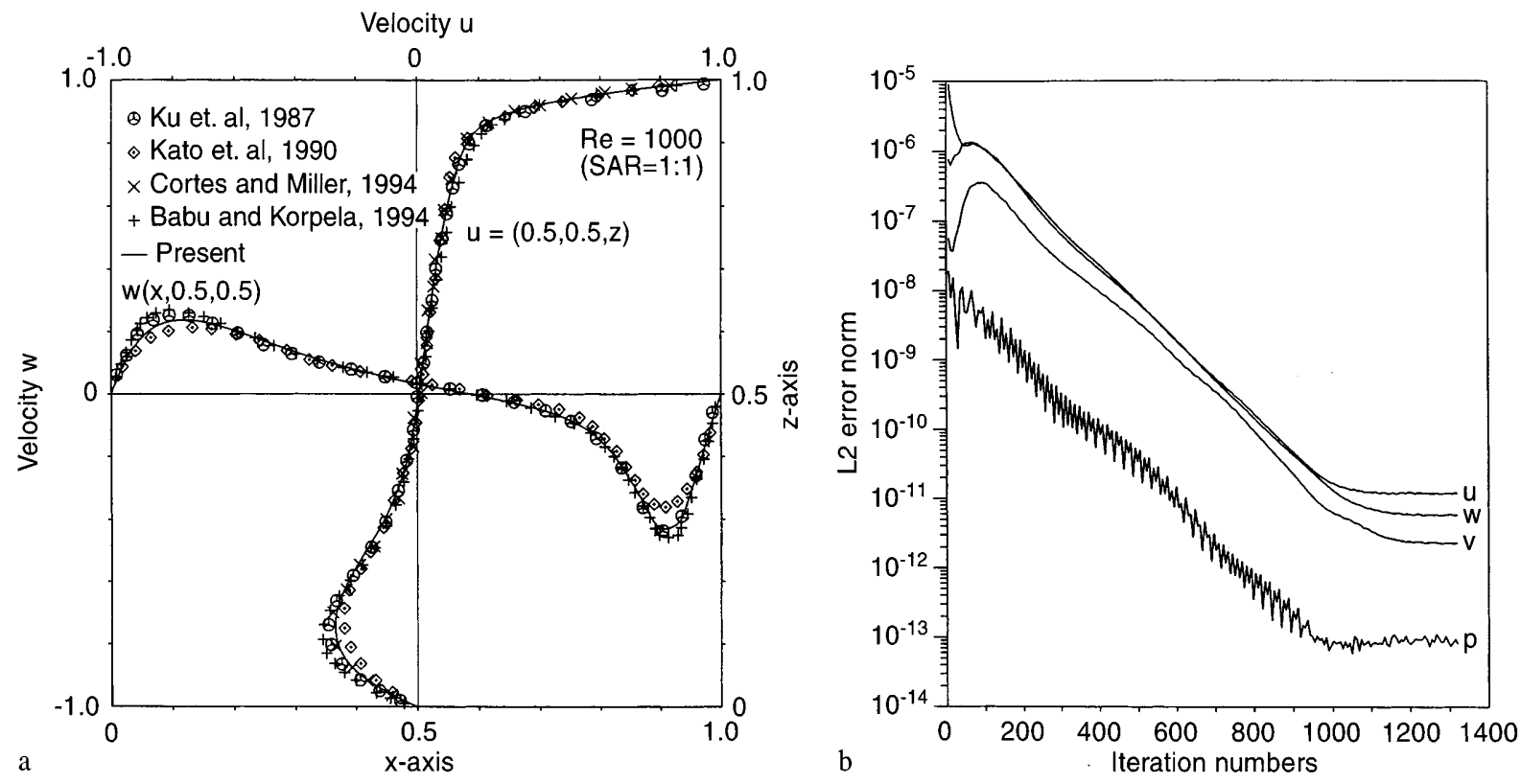

Fig. 5a,b. Comparison study for $\mathrm{SAR}=1: 1$ and $\mathrm{Re}=1000$. a mid-sectional velocities at the symmetry plane $y=0.5$; $\mathbf{b}$ error reduction for working variables
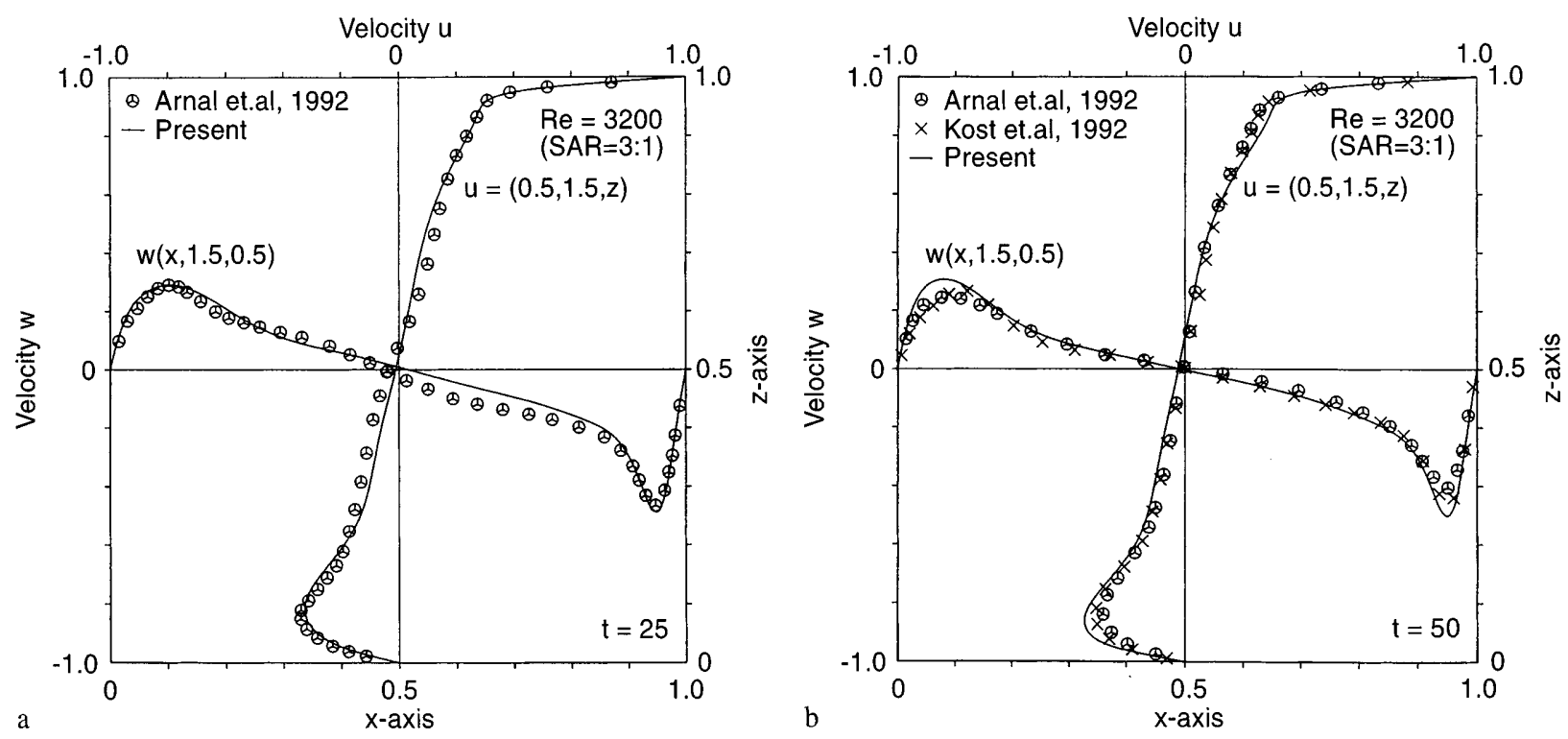

Fig. 6a,b. Comparison study for $\mathrm{SAR}=3: 1$ and $\mathrm{Re}=3200$. a mid-sectional velocities at the symmetry plane $y=1.5,(t=25)$; b mid-sectional velocities at the symmetry plane $y=1.5,(t=50)$

in the primary core, as seen in Fig. 7, which, in turn, results in a spanwise velocity moving towards the symmetry plane. Taking into consideration a control volume containing either one of the two end walls as part of its control surface, conservation of mass suggests the presence of the spanwise velocity with a sign which is opposite to that found in the core of the flow. This implies that fluid particles adjacent to the two side walls, the lid plane, and the floor of the cavity move towards the two end walls. To show that there is a sign switching of the spanwise velocity in the flow, we plot the zero contour of the spanwise velocity component $v$ in Fig. 7. Collecting lines of this sort at the $x$ planes results in the three-dimensional contour surfaces of $v=0$ in Fig. 8. This spanwise motion, together with an apparent circulation flow pattern at the spanwise plane, constitutes a helical vortex structure. With this spanwise velocity, which is typical of lid-driven cavity flow motion, the flow pattern at the $x$-plane takes the form shown in Fig. 3. Due to space considerations, we have not plotted velocity vectors at longitudinal planes other than the $x=0.525$ plane. As to the flow structure at the $z$ plane, it is virtually the same as that found at the $x$ plane. Interested readers are referred to our previous work (Chiang et al. 1996).

As Fig. 3 reveals, there is an apparent pair of so-called corner vortices. The formation of corner vortices 


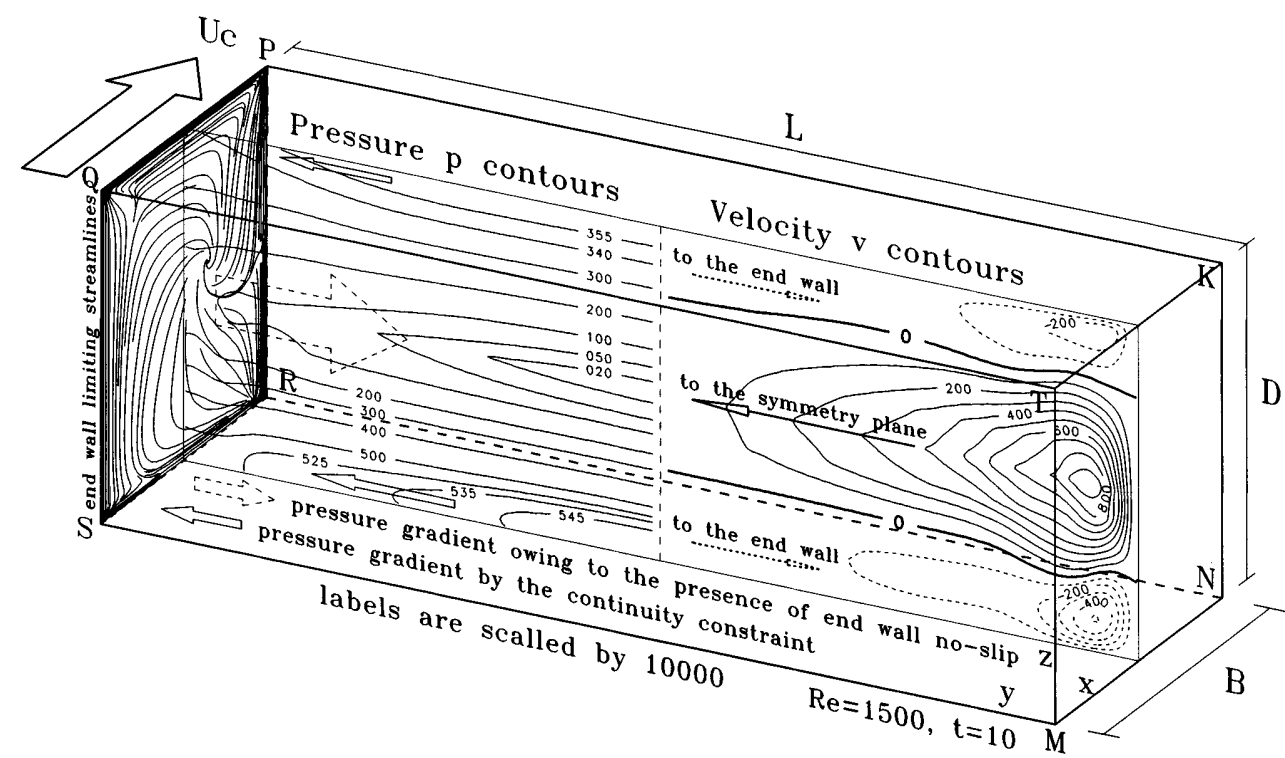

Fig. 7. The end wall induced pressure gradient, spanwise velocity, and the limiting streamlines constitutes the global transport mechanism in that fluid particles near the wall region are engulfed into the primary core with the aid of corner vortices. Corner eddies, as a consequence, aid the spanwise exchange of fluid particles in the cavity. This conceptually amounts to placing a suction pump on the end wall, to which the nearby particles are attracted. In support of our conjecture, we plot in Fig. 3 the limiting streamlines at a $y$-plane which is fairly close to the end wall, say at $y=2.99$. Notably, there is indeed an attracting spiral-saddle point at the $y=2.99$ plane, which is exactly where the suction pump is located.

Our computed solutions reveal that the flow field due to the lid motion asymptotically approaches a full developed state at a time around $t=25$. Subsequent to this time, particle motions, inside the tube of $v=0$ contour surface shown in Fig. 8, from the two ends meet at the symmetry plane. This opposite spiraling motion causes the contour surface of $v=0$ to distort and the size to enlarge. On increase of time, to respond this variation TolluniemSchlichting wave emerges from the symmetry plane and propagates towards the two end walls. This phenomenon is particularly apparent at the upstream side of the cavity. Finally, Taylor Görtler like vortices burst at time $t=85$. The main objective of the rest of the discussion will be directed towards answering why, where, and how Taylor Görtler vortices emerge.

\section{3}

\section{Secondary eddies}

Examining the literature, it seems that studies on the liddriven cavity problem have mostly concentrated on the primary core and the downstream secondary eddy. Very few or none of them have been devoted to study of the upstream secondary eddy. Consequently, our attention is now directed towards the upstream secondary eddy. Recall that fluid particles spiraling towards the end wall tend to be pulled into the primary core by the corner vortex. These engulfed fluid particles through the singular point (or attracting spiral saddle point) spiral quickly and monotonically towards the symmetry plane. This provides a means of destabilizing the flow in the cavity. With this in mind, we will attempt to show that the upstream secondary eddy is less stable, as compared to its downstream secondary eddy.

To support our viewpoint, we have marked the particles which were originally located at the USE and DSE and traced their Lagrangian-particle paths in Fig. 9. For illustration purposes, the projections of these particle paths onto the $x-y$ plane are also given in Fig. 9. Once particles within the secondary eddies spiral towards the end wall, they are engulfed into the primary core through the corner vortices. This is followed by a spiraling particle motion, which is characterized by quick and monotonic migration towards the symmetry plane, in the upstream secondary eddy. On the other hand, particles from the downstream secondary eddy spiral back-and-forth towards the symmetry plane. In view of the difference in the nature of the spiraling particles which migrate towards the symmetry plane, we can see clearly that the flow in the upstream side wall is more unstable. As compared with the location where the particle in the secondary eddy is engulfed into the primary core, we can clearly see that particles in the USE are drawn into the interior of the cavity, as seen in Fig. 9b. Particle paths for the DSE fluid particles are, on the other hand, seen in the vicinity of the $v=0$ contour surface shown in Fig. 9a. Another interesting finding which needs to be addressed is that the nearer the engulfed particle approaches the end wall, the higher the possibility is that a fluid particle can be drawn in the primary core through the attracting spiral node. Confirmation of this finding is best illustrated in Fig. 9c. It is this back-andforth spiraling motion for the particle seeded in the downstream secondary eddy which aids flow stability.

Now, we will show how a spiraling particle seeded in the USE is engulfed into the core differs from a particle in the DSE. To begin with, it is worthwhile to present evidence that at a spanwise plane which is sufficiently distant from the end wall, say at the plane $y=2.8$ shown in Fig. 10a, the streamline pattern is akin to that computed from the twodimensional analysis. Three lines labeled "a", "b", and "c" 

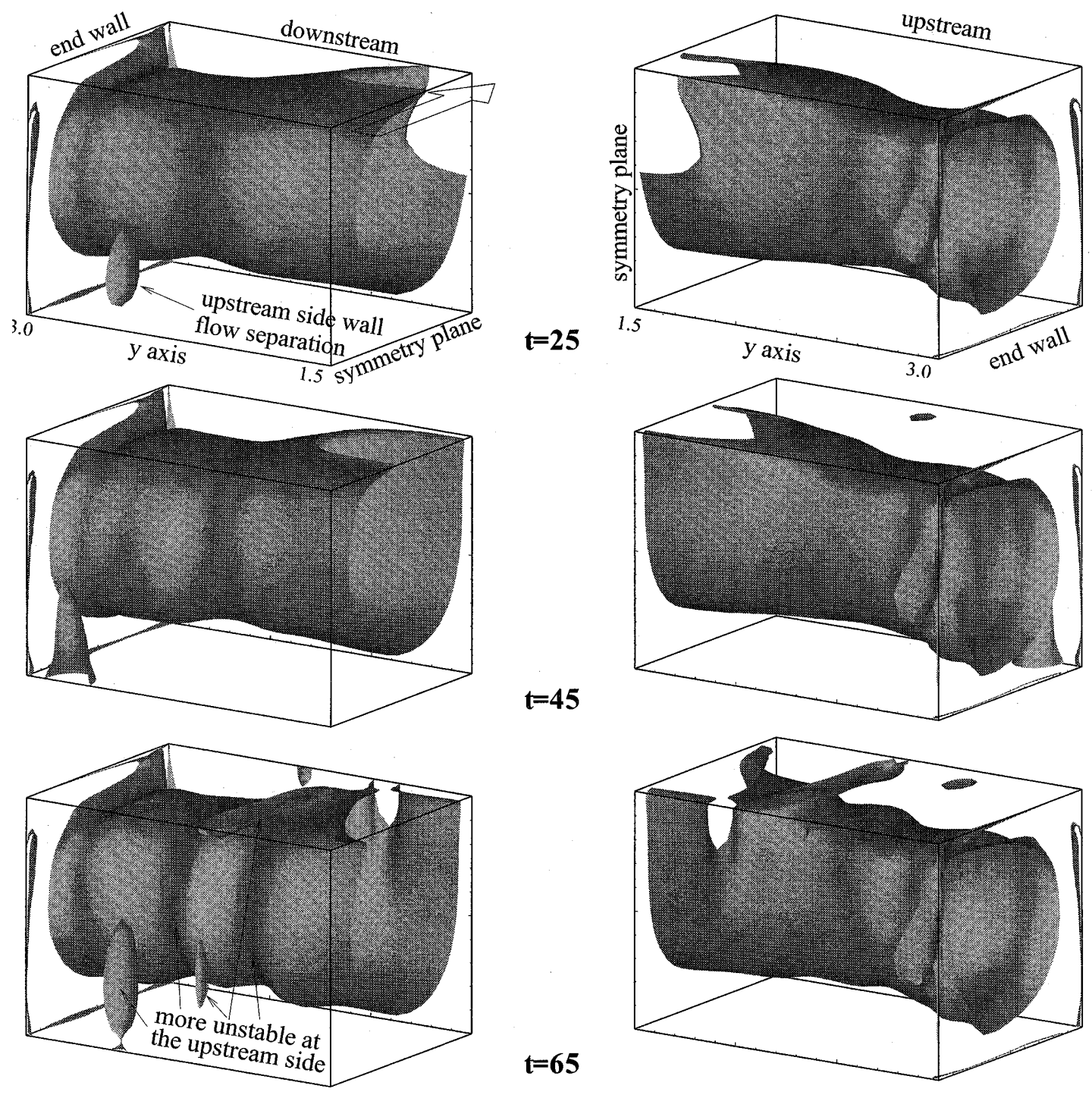

$\mathbf{t}=65$
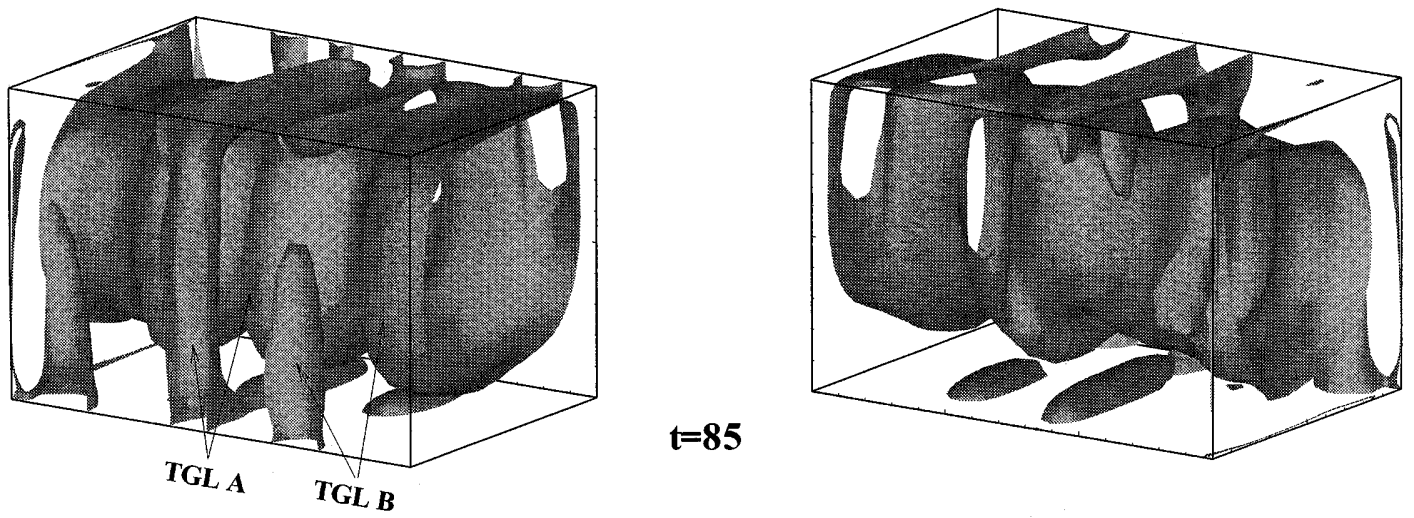

b viewing from the downstream side

\section{a viewing from the upstream side}

Fig. 8a,b. Three-dimensional $v=0$ isosurfaces in a half-span cavity $1.5 \leq y \leq 3$ plotted at several selected times $t=25,45,65,85$. a viewing from the upstream side; $\mathbf{b}$ viewing from the downstream side

in Fig. 10a are useful to reveal how particles proceed towards the secondary eddies and the primary core. It is interesting to point out that, within the primary core, particles inside/outside of the $v=0$ contour lines spiral outwards in their migration to the symmetry/end wall, respectively. As the end wall is approached, the downstream secondary eddy at the $y=2.9$ spanwise plane, as shown in Fig. 10b, breaks. The spiraling particle motion within the primary core starts to spiral inwards. This paves the way for the particles inside the DSE to be engulfed into 


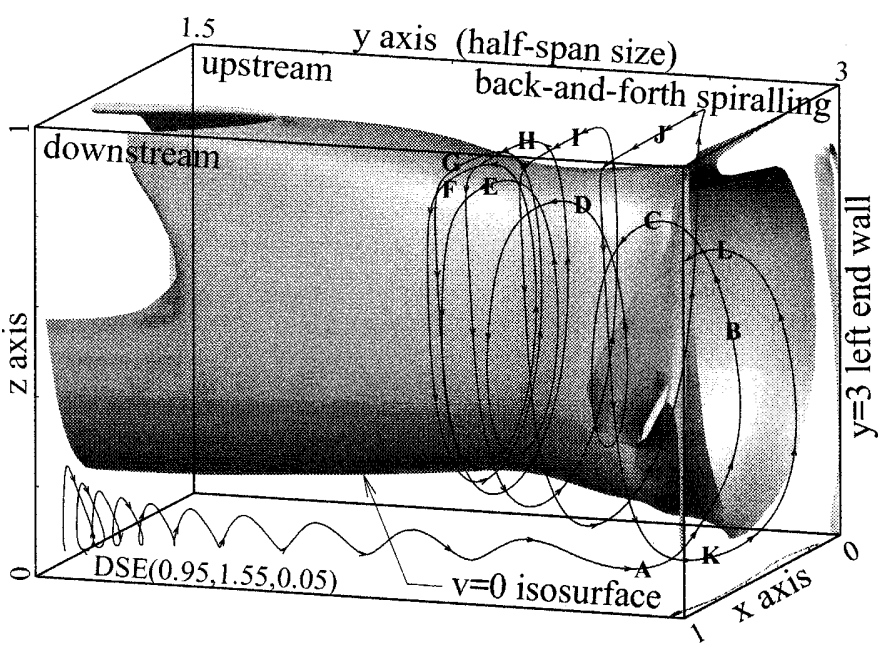

$\mathbf{a}$

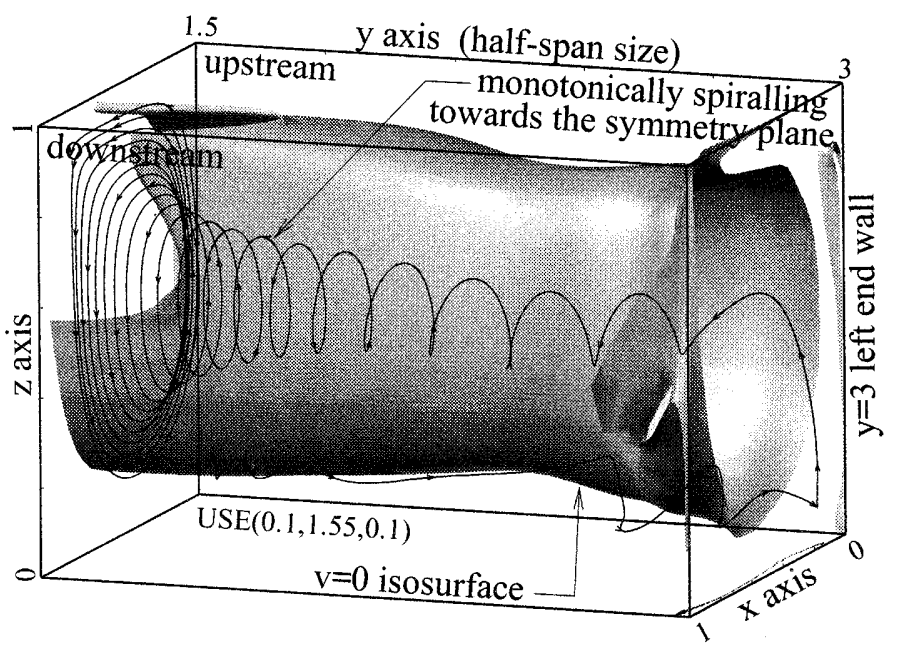

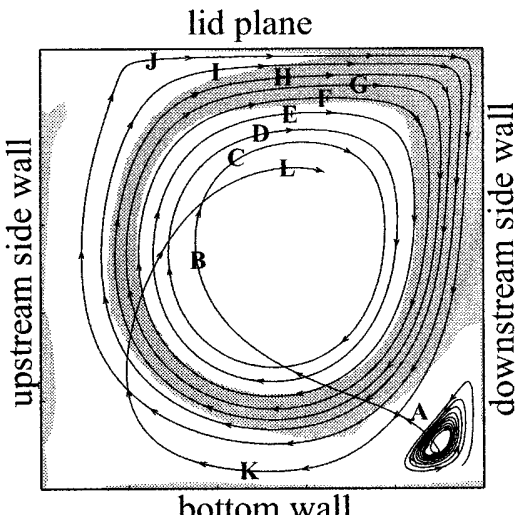

endwall attracting: $A-B, K-L$ forth spiralling: C-D-E-F back spiralling: G-H-I-J

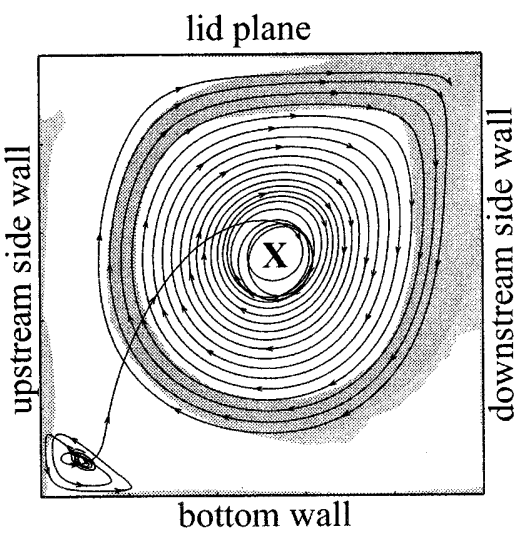

$\mathbf{X}$ - Spiral-Saddle at the end wall

b

particle spiralling in the USE

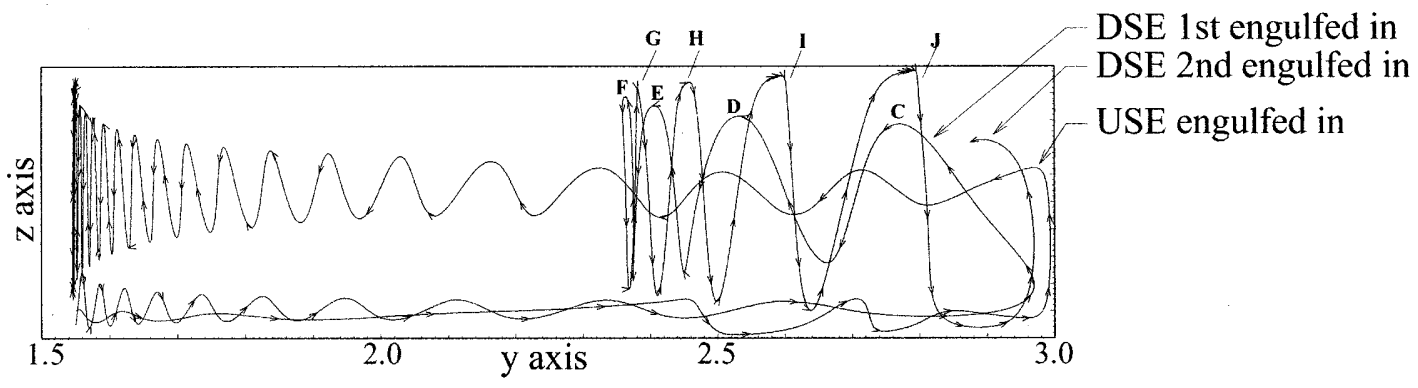

c

comparison on the endwall suction

Fig. 9a-c. Illustration of spiraling particles at $t=25$. a spiraling particle in the DSE; b spiraling particle in the USE; c comparison of particle paths for particles, originally inside secondary eddies, which have been engulfed into the primary core via the corner vortex

the primary core. The plane at $y=2.9$ is still too far away from the end wall for the fluid particles to be engulfed into the primary core. At the spanwise plane which increasingly approaches the end wall, the upstream secondary eddy at the spanwise plane $y=2.925$ shown in Fig. 10c also breaks. Particles inside the upstream secondary eddy begin to be sucked into the primary core.
Particles at spanwise planes which are fairly close to the end wall, for example at the two planes shown in Figs. 10c and $\mathrm{d}$, are characterized as having a tendency to be entirely engulfed into the attracting spiral-saddle. According to the computed solutions, we can see that the particle inside the downstream secondary eddy is engulfed into the primary core before that in the upstream secondary eddy is. 

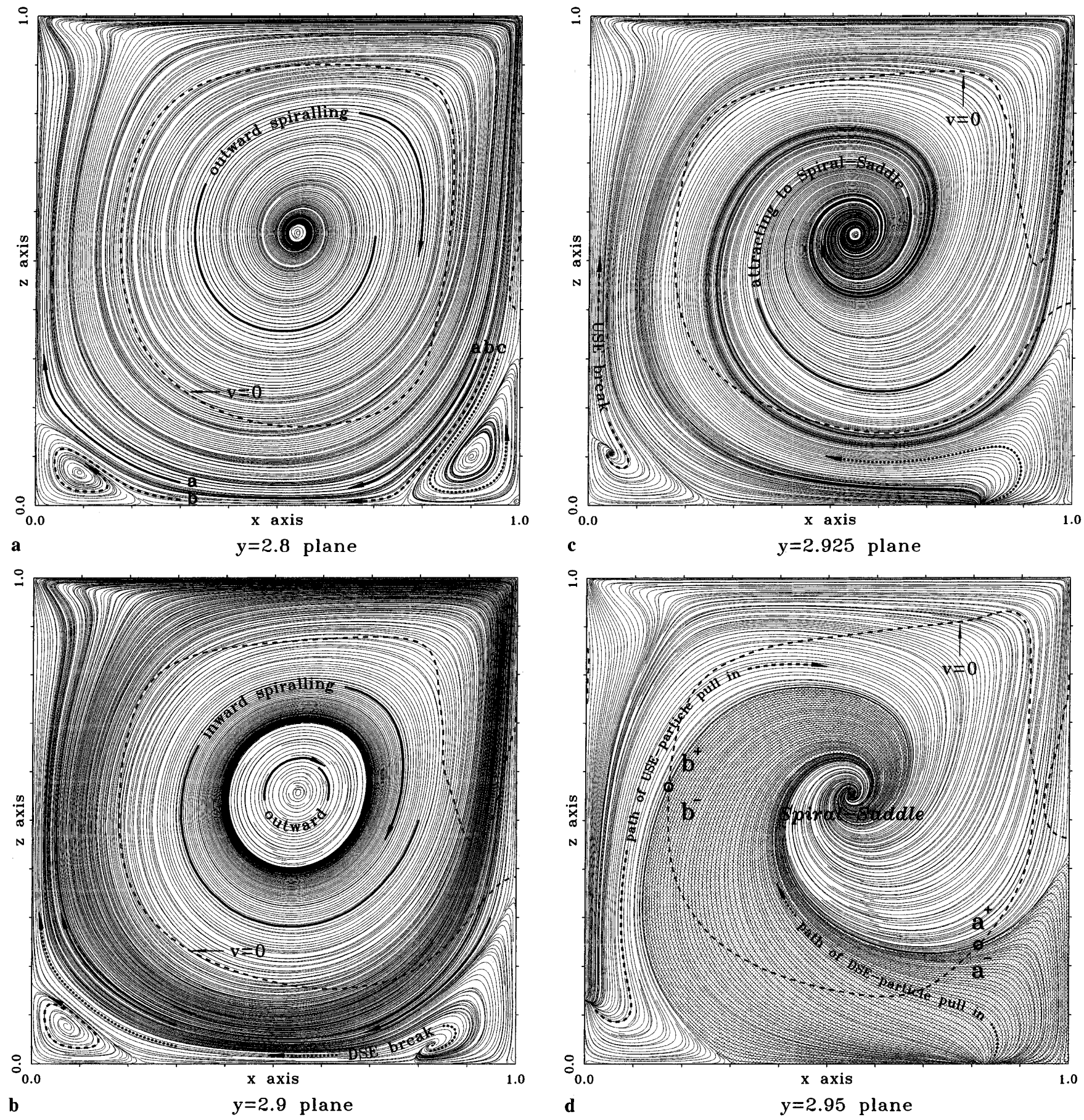

Fig. 10a-d. Description of spiraling processes at planes near the end wall by streamline plots at $t=25$. $\mathbf{a} y=2.8$ plane; $\mathbf{b} y=2.9$ plane; c $y=2.925$ plane; $\mathbf{d} y=2.95$ plane

According to Fig. 10d, particles in the downstream secondary eddy are engulfed into the $v=0$ contour line through the line labeled by $\overline{a^{-} b^{-}}$while particles inside the upstream secondary eddy are attracted into the spiralsaddle node via the other part of $v=0$ contour line, namely $\overline{a^{+} b^{+}}$. Recall that particles inside the upstream secondary eddy can be drawn in the primary core only at a spanwise plane which is fairly close to the end wall. Once the upstream secondary eddy is broken, particles inside this eddy are mostly engulfed into the primary core via the spiralsaddle. These particles will migrate quickly and monoton- ically towards the symmetry plane in regions adjacent to the vortical core line, which is the collection of spiral-saddle points computed at different spanwise planes. Bearing in mind that the engulfed particles from the upstream secondary eddy tend to destabilize the flow, under normal conditions, universal law has a tendency to keep the flow system in its most stable status. This suggests that the supply of particles to the upstream secondary eddy is prohibited so as to keep the flow system stable. In the direction from $y=1.8$ (see Fig. 11a) to $y=2.1$ (see Fig. 11b), the entry to the upstream secondary eddy is prohibited so 


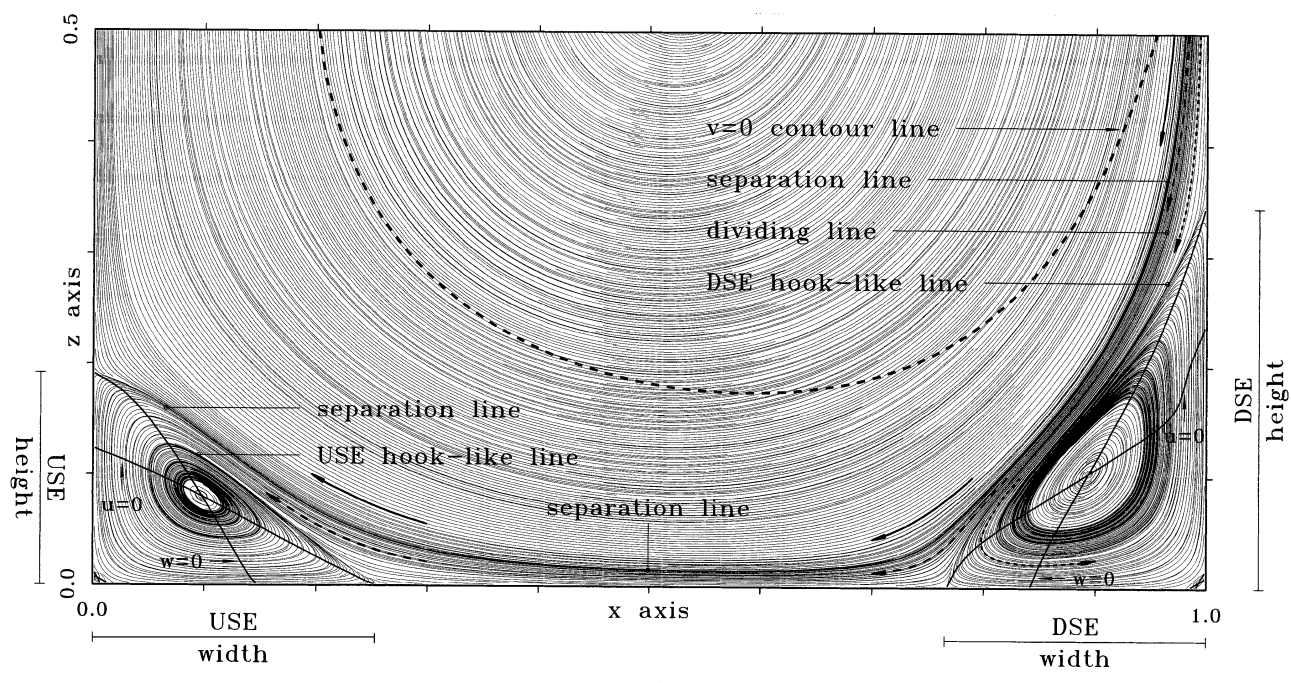

$\mathbf{a}$

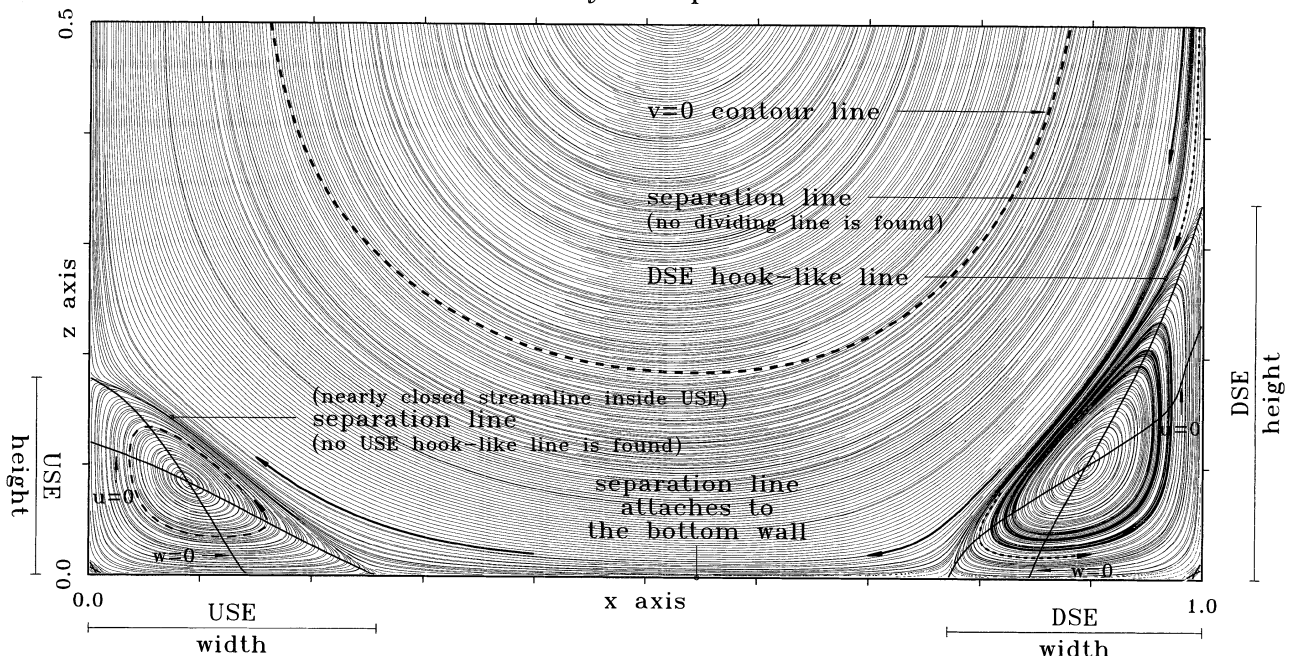

b

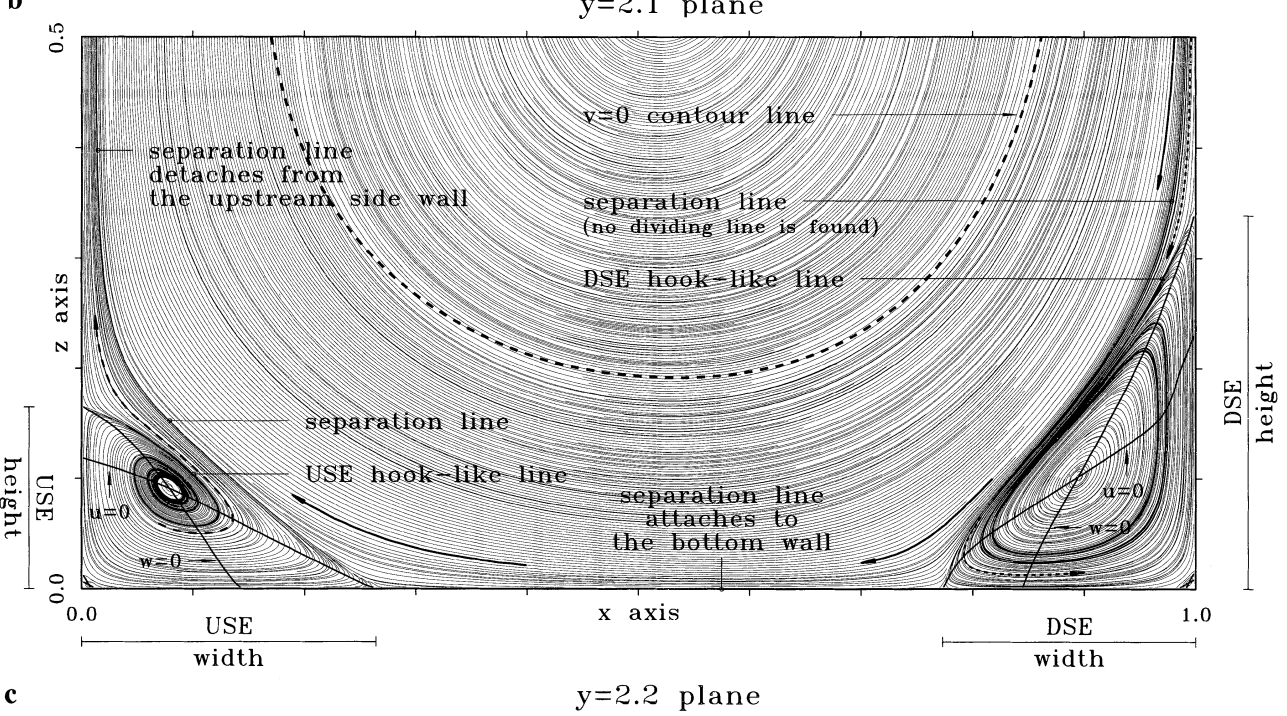

Fig. 11a-c. Computed streamlines at $t=25$ which illustrate how fluid flows exchange between the primary eddy and DSE/USE. a at $y=1.8$ plane; $\mathbf{b}$ at $y=2.1$ plane; $\mathbf{c}$ at $y=2.2$ plane as to yield a more stable flow condition. This is followed by the break of the upstream secondary eddy at a plane $(y=2.2)$ (see Fig. 11c) which is not closer enough to the end wall. This series of changes just mentioned is best described by the so-called separation lines plotted in Fig. 11.
4.4

\section{Particle exchange among eddies}

Prior to discussing the time history of the spanwise transport phenomena, it is instructive to plot streamlines at each $y$ plane so that the structure of the particle motion 
can be clearly illustrated. As shown in Fig. 11a, we define several lines of different physical relevance for descriptive purposes. The line, which is farthest away from the two bottom corners, represents the separation line, above which the flow domain is referred to as the primary core region. The line between the separation line and the downstream side wall is referred to as the dividing line, which divides two streams entering into downstream and upstream secondary eddies. Fluid particles confined to the regions of plotted helixes proceed spirally to the end wall.

Due to space considerations, we will describe the transport phenomena at the spanwise $y$-planes based mainly on the finite volume solutions computed at $t=25$. In a half domain, $1.5 \leq y \leq 3$, Fig. 12 shows several possibilities for particles to exchange between the downstream side and its upstream counterpart. In the range of $1.5<y<2.1$, fluid particles near the upper downstream side wall can enter into upstream and downstream secondary eddies. As the $y=2.1$ plane is approached, entry into the USE is prohibited. In between $2.1 \leq y \leq 2.55$, fluids coming from the upper half of the cavity enter entirely into the DSE and then proceed to the end wall in a spiraling manner. In this spanwise range, which has a length of 0.45 , fluid particles below the separation surface as well as at the downstream side have no chance of communicating with particles at the upstream side. At spanwise planes in between $y=2.55$ and $y=2.85$, the transfer processes repeat. As the end wall is further approached, a remarkable change is observed. Fluid flows inside the downstream secondary eddy can be directly sucked into the core from the zero spanwise velocity contour lines of $\overline{a^{-} b^{-}}$shown in Fig. 10d. As to fluid particles in the upstream secondary eddy, they are lifted up and are then entrained into the primary core from $\overline{a^{+} b^{+}}$. For completeness, we have plotted $v=0$ contour lines, separation lines, dividing lines, and DSE/USE hooklike lines at different spanwise locations and different times, $t=25$ and 85, in Figs. 12-13, respectively.

Once entry into the upstream side is prohibited, fluid flow inside the upstream secondary eddy is forcibly sucked into the primary core. This accompanies with the detachment of the separation line from the upstream side wall. The upstream secondary eddy breaks at this moment. In the vicinity of the two end walls, Fig. 13 shows that fluid particles inside the secondary eddy but outside of the contour line of $v=0$ are all drawn into the core.

As the end wall is approached, the configuration of the separation line and, thus, its curvature undergoes a change. Since the separation line which bounds the primary core is analogous to the concave wall over which there is a laminar flow, the change in the curvature of the separation line results in variation of exerted centrifugal force. As the separation line is detached from the upstream side wall, the resulting curvature is sufficiently appreciable to cause force imbalance between the viscous and centrifugal forces. Taylor-Görtler-like vortices are the direct result of this force imbalance due to the appreciable variation in the centrifugal force. We will discuss the formation of TGL vortices in more detail later.

\section{5}

\section{Eddy structure and formation of TGL vortices}

Near the downstream side wall, particles located at a spatial location with a larger value of $z$ may enter into the upstream or downstream secondary eddies (see Fig. 11a). Particles inside the upstream secondary eddy may be drawn upward and, thus, pulled out of this secondary eddy (see Fig. 11c). To provide a clear picture which confirms this statement, it is instructive to integrate separation lines, plotted in Figs. 12-13, at different $y$-planes for the time in the range of $t=25 \sim 85$. The resulting three-dimensional curved interfaces exclusively separate the primary core from the secondary eddies. Due to space considerations, we only plot them at some representative times in Figs. 14a-17a. These include a time when the primary flow has reached a well developed state $(t=25)$, and subsequent times $(t=45,65)$, and the time when TGL vortices emerge $(t=85)$.

To explore in depth the transport phenomena in the full cavity, it is important to shed light on the mechanism leading to the exchange of flow between primary and secondary eddies. Figure 14-17 depict different views of separation surfaces, which reveal how the curvature of the primary flow motion changes. As seen in Figs. 14-17, the change in the radius of the curvature along the spanwise direction has a great influence on the onset of laminar instabilities. Such instability is closely associated with the centrifugal force. Clearly visible on the separation surface are several localized troughs, across which force balance can adjust itself. As these figures show, the separation surface at the upstream side is much more irregular than is that at the downstream side. In crossing over a narrow trough, the primary flow at the upstream side definitely experiences a larger change of the centrifugal force, as compared with that on the downstream side. We interpret this marked variation of the curvature on the separation surface as being the main cause leading to the subsequent emergence of TGL vortices.

To support our claim that the flow near the upstream side wall is more unstable than its downstream counterparts, we will present evidence from different viewpoints. In view of the fact that the separation surface detaches locally from the upstream side wall, as indicated by " $\alpha$ " in Fig. 14, " $\alpha$ " and " $\beta$ " in Fig. 15, " $A$ ", " $\alpha \beta$ " and " $\gamma$ " in Fig. 16, and " $B$ ", “ $A$ ", “ $\alpha \beta \gamma$ " in Fig. 17, fluid particles inside the upstream secondary eddy no longer spiral towards the two end walls. These particles are rather locally pulled out of the upstream secondary eddy and entrained directly into the primary core. The physics explaining how fluid particles inside the upstream secondary eddy communicate with those in the primary core are clearly demonstrated by the particle tracer plots in Figs. 14b-17b. For the sake of completeness, we summarize in Fig. 18 the starting and terminating spanwise locations of the separation surface, which detaches from the upstream side wall. It is important to note that the wrinkles, as found in Figs. 14-17, along the spanwise direction correspond to the varied sizes of the USE and DSE plotted in Fig. 19, and to the $v=0$ contours plotted in Fig. 8 . 

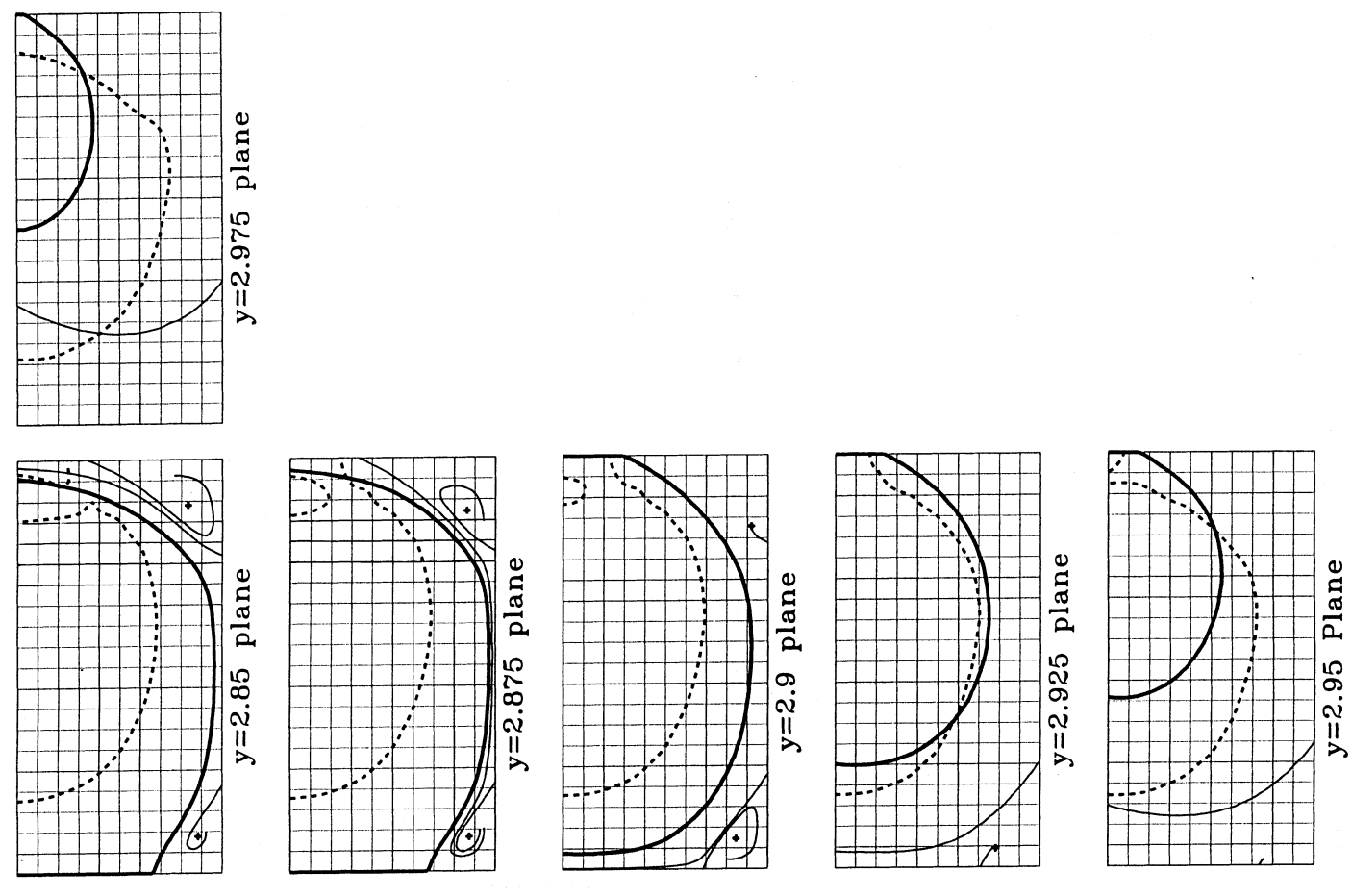

$\stackrel{2}{4}$
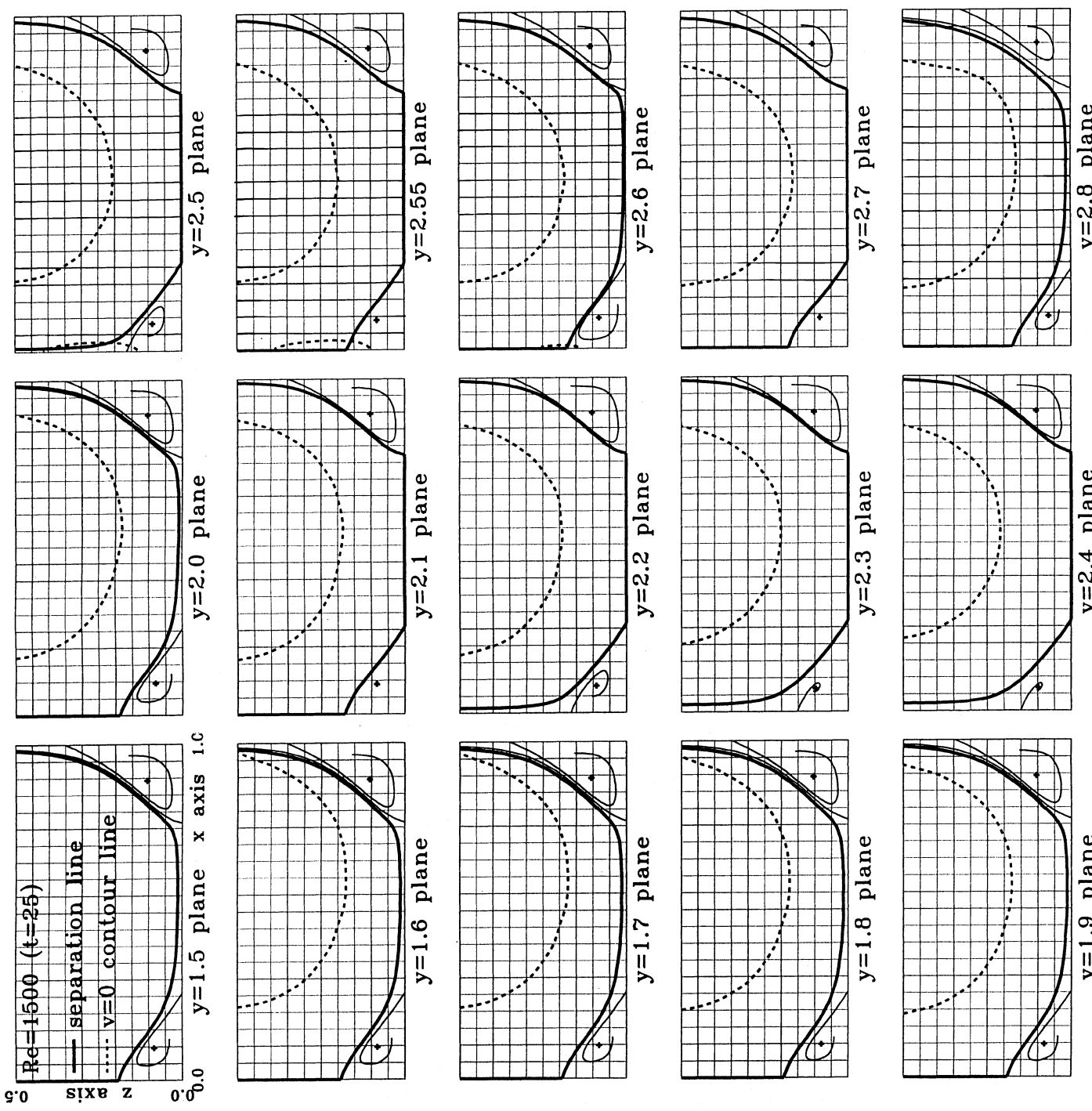

$\stackrel{\Xi}{\Xi}$ 


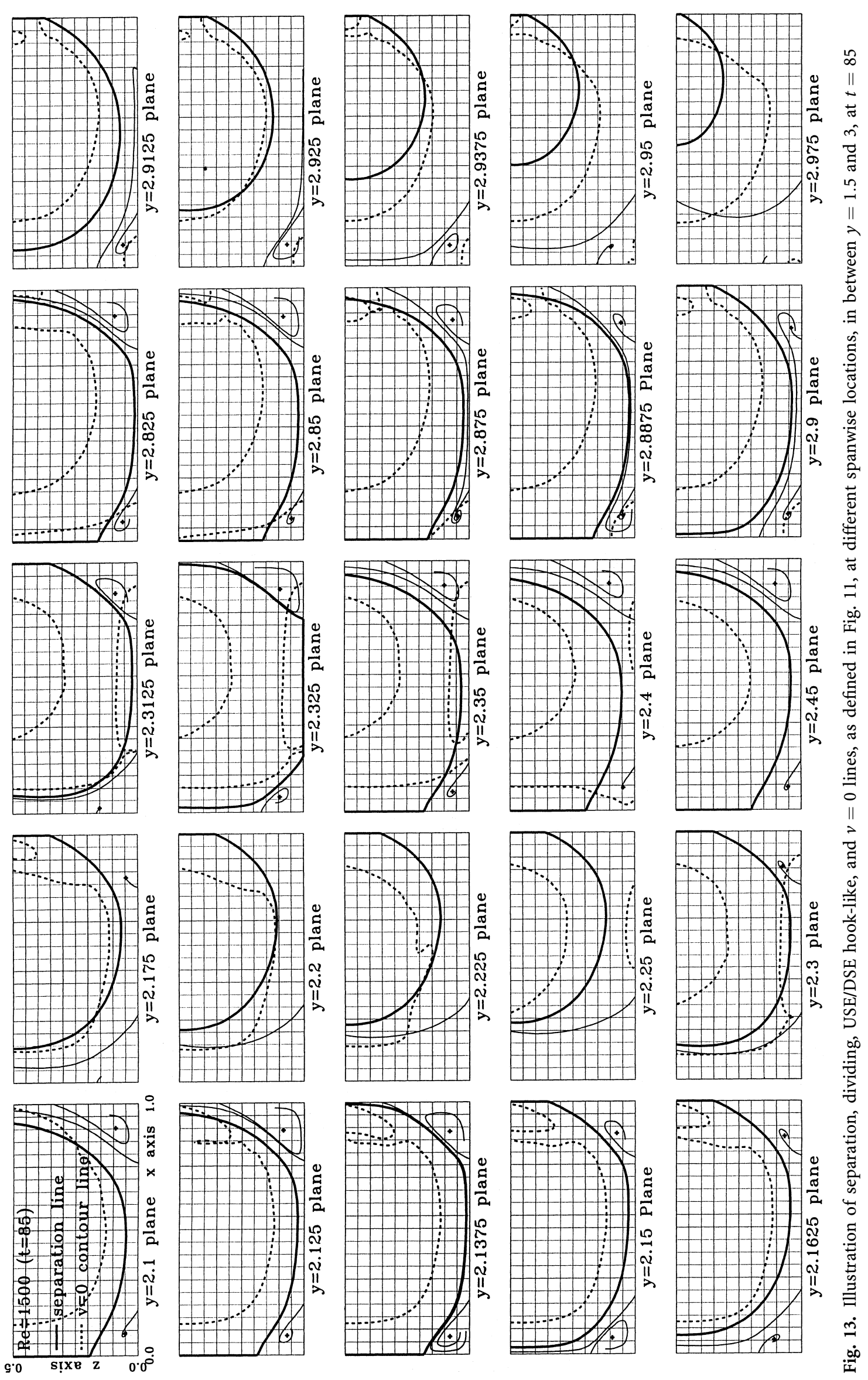




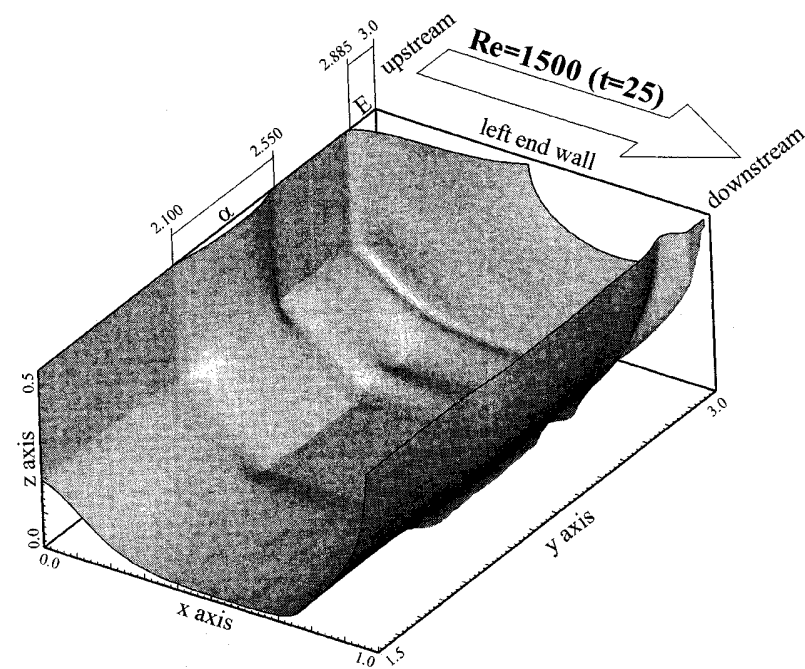

a

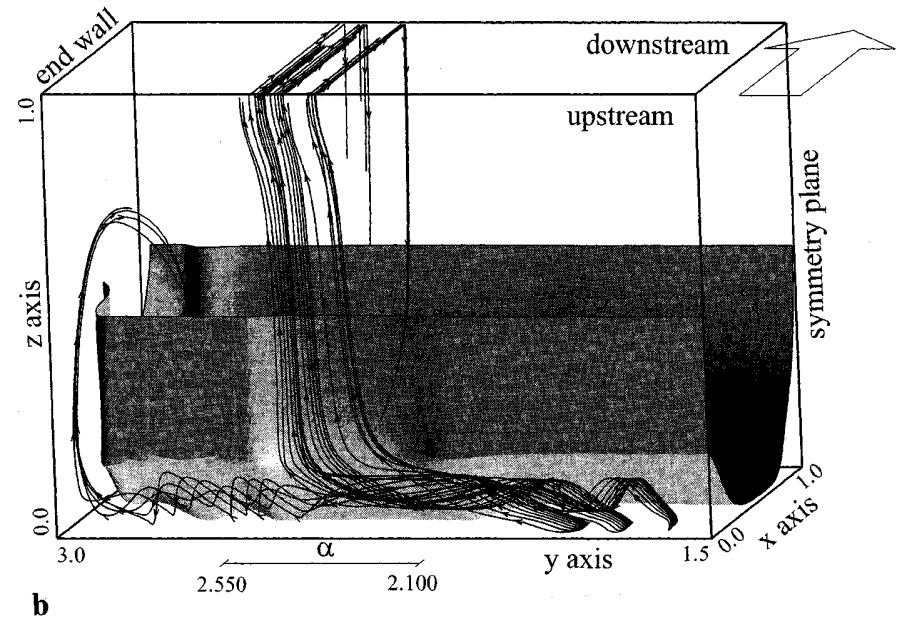

Fig. 14. A plot showing that at $t=25$ the separation surface may detach from the upstream side wall and the end wall (a). Fluid particles inside USE may enter into the primary core (b)

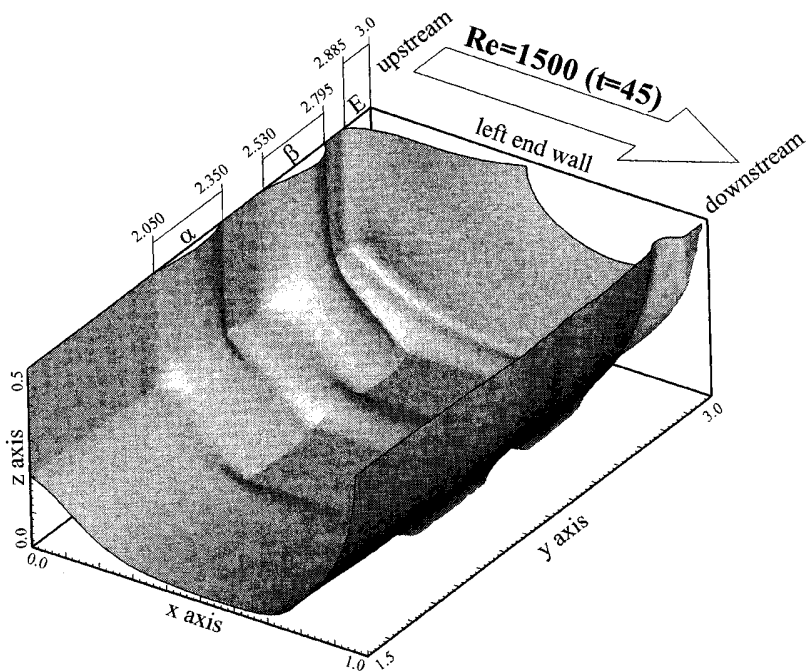

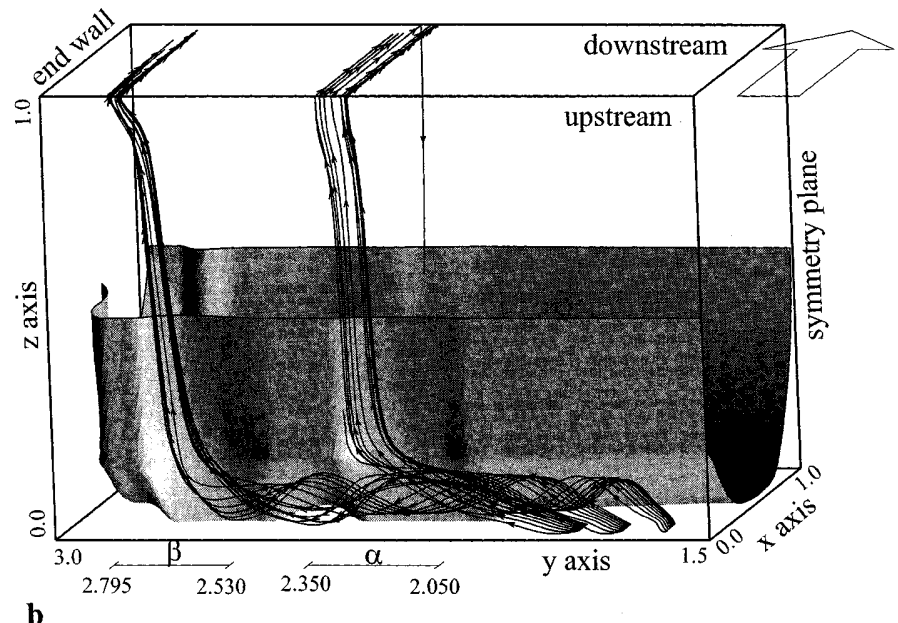

b

Fig. 15. A plot showing that at $t=45$ the separation surface may detach from the upstream side wall and the end wall (a). Fluid particles inside USE may enter into the primary core $(\mathbf{b})$

On the upstream side wall, we designate in Fig. 18 the dotted region as the disturbance region. Thanks to the formation of this upstream disturbance region, the resulting upstream detachment allows fluid particles to be locally entrained to the primary flow motion. This aids flow stability in that fewer fluid particles will be sucked into the primary core from regions near the end wall and then spiral monotonically towards the symmetry plane. Besides the end-wall detached separation surfaces and the region marked by " $E$ ", as shown in Figs. 14-17, there exists only one detachment region " $\alpha$ " at time $t=25$, followed by an additional detachment region " $\beta$ " in between " $\alpha$ " and "E" at time $t=45$. Subsequent to $t=45$, detachment cells " $\alpha$ " and " $\beta$ " merge together and induce a smaller detachment cell, " $\gamma$ ", in between " $\alpha \beta$ " and " $E$ ". At a later time $t=65$, a new detachment cell " $\mathrm{A}$ " emerges in front of " $\alpha \beta$ ". This paves the way for the formation of TGL vortices, as depicted in Fig. 20, at time $t=85$.

Recall that the contour surface of $v=0$, as shown in Fig. 8, will be distorted in the course of flow evolution. Across this surface there is a change in the spanwise velocity direction, resulting in vortex formation. As seen in Fig. 20, this vortex is formed at a location near the $v=0$ contour surface which is sufficiently distant from the floor of the cavity. Since this vortex is irrelevant to the fluid viscosity, we refer to this vortex as a free-shear vortex. To ensure the existence of the free-shear vortex, the pressure field is established. This will increasingly influence the spiraling fluid flow in the sense that the boundary layer flow moving towards the end wall will separate from the floor of the cavity. A wall-shear vortex is thus formed in response to the compression of the free-shear vortex to the 


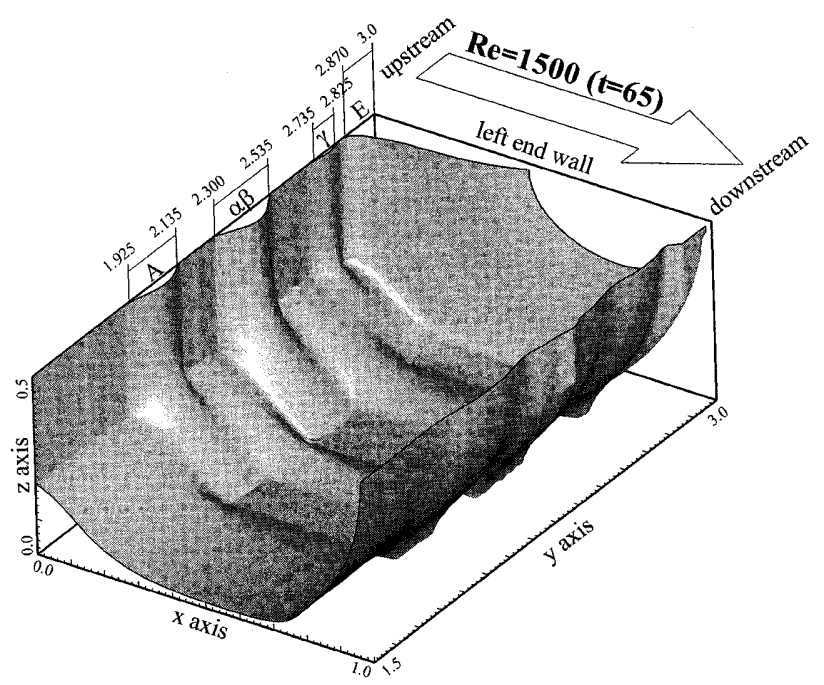

a

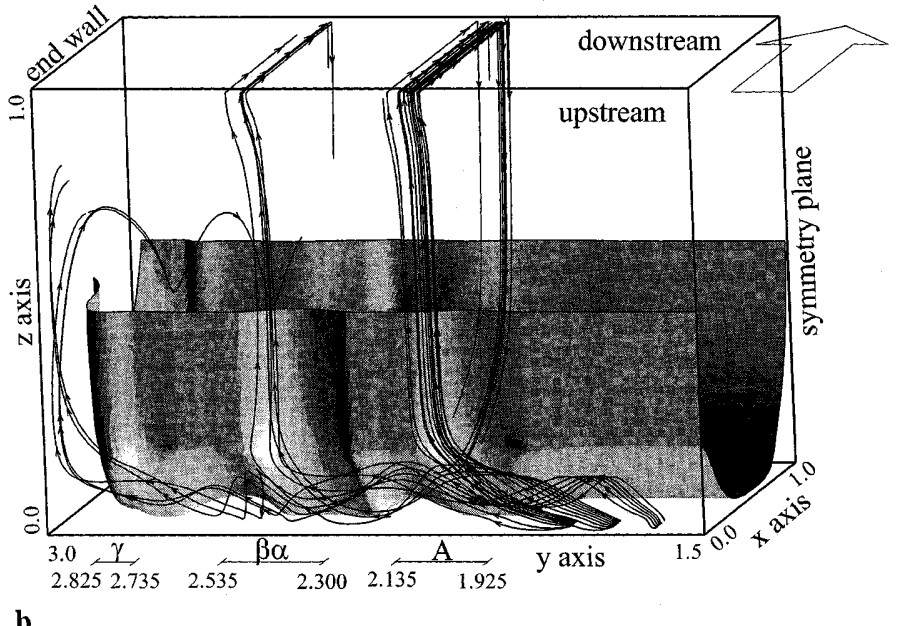

b

Fig. 16. A plot showing that at $t=65$ the separation surface may detach from the upstream side wall and the end wall (a). Fluid particles inside USE may enter into the primary core (b)
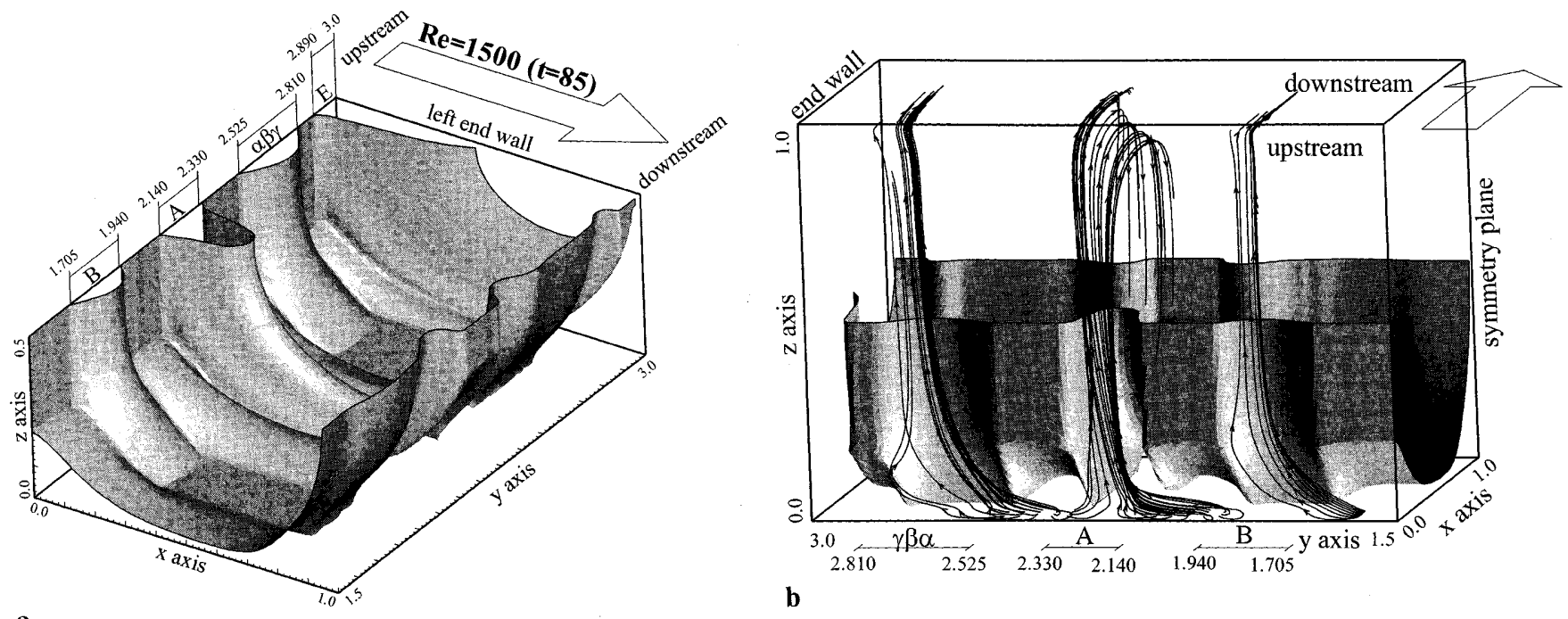

Fig. 17. A plot showing that at $t=85$ the separation surface may detach from the upstream side wall and the end wall (a). Fluid particles inside USE may enter into the primary core (b)

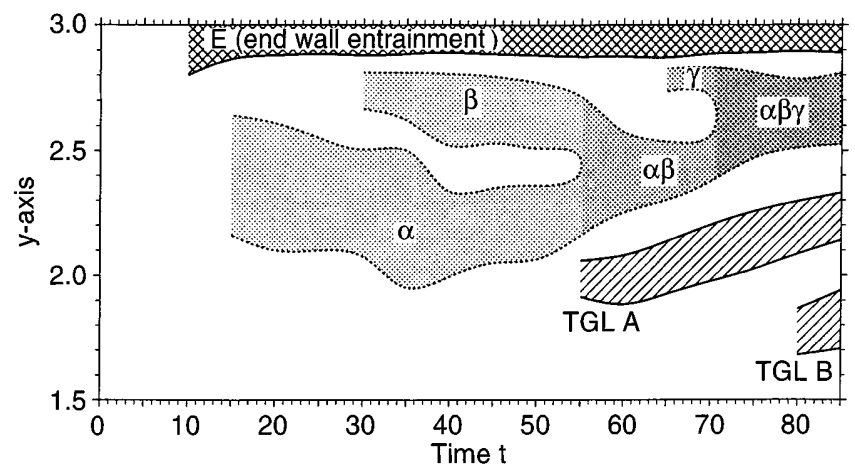

Fig. 18. Description of the time history of the size of the detached upstream separation surface measured in $y$ floor of the cavity. Vortices of two different kinds gradually approach each other and form so-called Taylor Görtler like vortices at $t=85$, as seen in Fig. 3 . Due to space considerations, the subsequent transport of at most five pairs of TGL vortices is not described in this paper. Interested readers may refer to (Chiang et al. 1996) for additional details.

\section{5 \\ Concluding remarks}

While the geometry of the cavity examined here is extraordinarily simple, the flow physics in the cavity are nevertheless rich. The physical complexity is attributable to the eddies which are characterized as possessing 

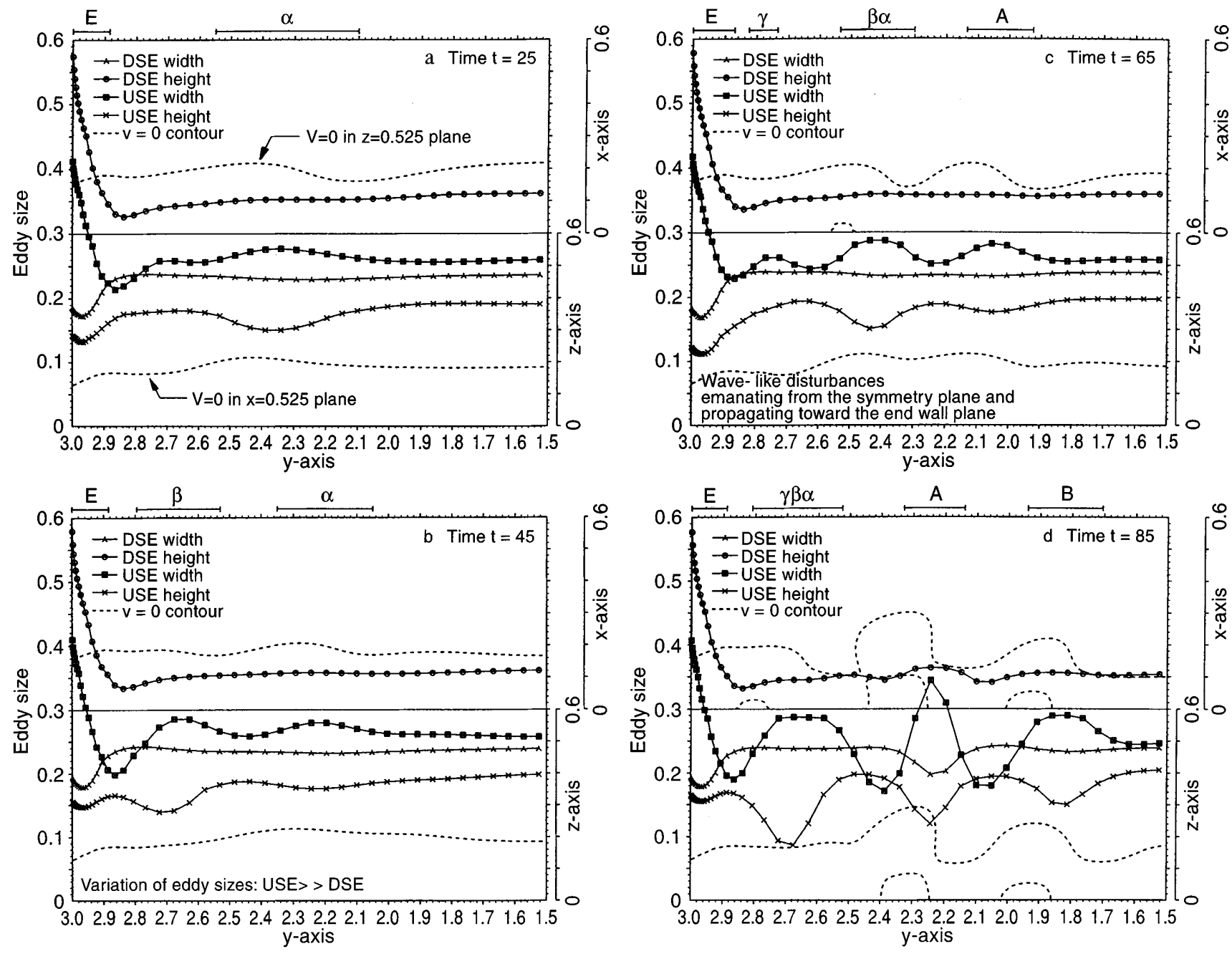

Fig. 19a-d. Illustration of the width and height of secondary eddies (defined in Fig. 11) and the contour lines of zero spanwise velocity at the $x=0.525$ and $z=0.525$ planes. a $t=25 ; \mathbf{b} t=45 ; \mathbf{c} t=65 ; \mathbf{d} t=85$

different sizes and characteristics. Also, how interaction proceeds among the eddies is crucial to the development into laminar instabilities. In the entire flow evolution, the transport mechanism is rooted largely in the spiraling nature of the flow motion established inside the secondary eddies and, of course, in the primary core. According to the finite volume solutions obtained, we conclude this paper with some important findings from this numerical simulation.

The three-dimensional lid-driven cavity flow is manifested by the presence of a spanwise velocity component which arises due to the presence of two vertical end walls. Accompanying the spanwise motion, the flow exhibiting the dominant recirculation flow pattern is prone to spiral. We interpret the presence of USE particles, which are engulfed from regions fairly near the two end walls into the primary core and then spiral monotonically towards the symmetry plane, as being the main cause leading to the flow instability because the two flow streams moving in opposite directions tend to collide with each other at the symmetry plane. This instability causes the surface separating the primary core and the upstream secondary eddy to detach from the upstream side wall. It is this distorted detachment which disrupts the well-balanced force between the centrifugal and pressure-gradient forces established inside the primary recirculating cell. This paves the way for the onset of Taylor-Görtler vortices.

Prior to describing the appearance of TGL vortices, we have studied in detail how eddies of different sizes and attributes are intimately coupled. This permits a systematic approach to understanding the complex interaction among spiraling eddies. As the end wall is approached, particles in the downstream secondary eddy begin to be engulfed into the primary core. This is followed by suction of particles in the upstream secondary eddy, which is closer to the end wall, into the primary core through the spiral-saddle point. The separation surface plotted in this paper furthermore helps to show that fluid flows present in the narrow wavy trough of the separation surface have a higher propensity to develop into TGL vortices. There exists a higher possibility that instabilities will result at spatial locations where the width of the upstream secondary eddy becomes appreciably larger than the width of the downstream secondary eddy. Computational experience from this study reveals that the size of the upstream secondary eddy, the configuration of the $v=0$ contour 

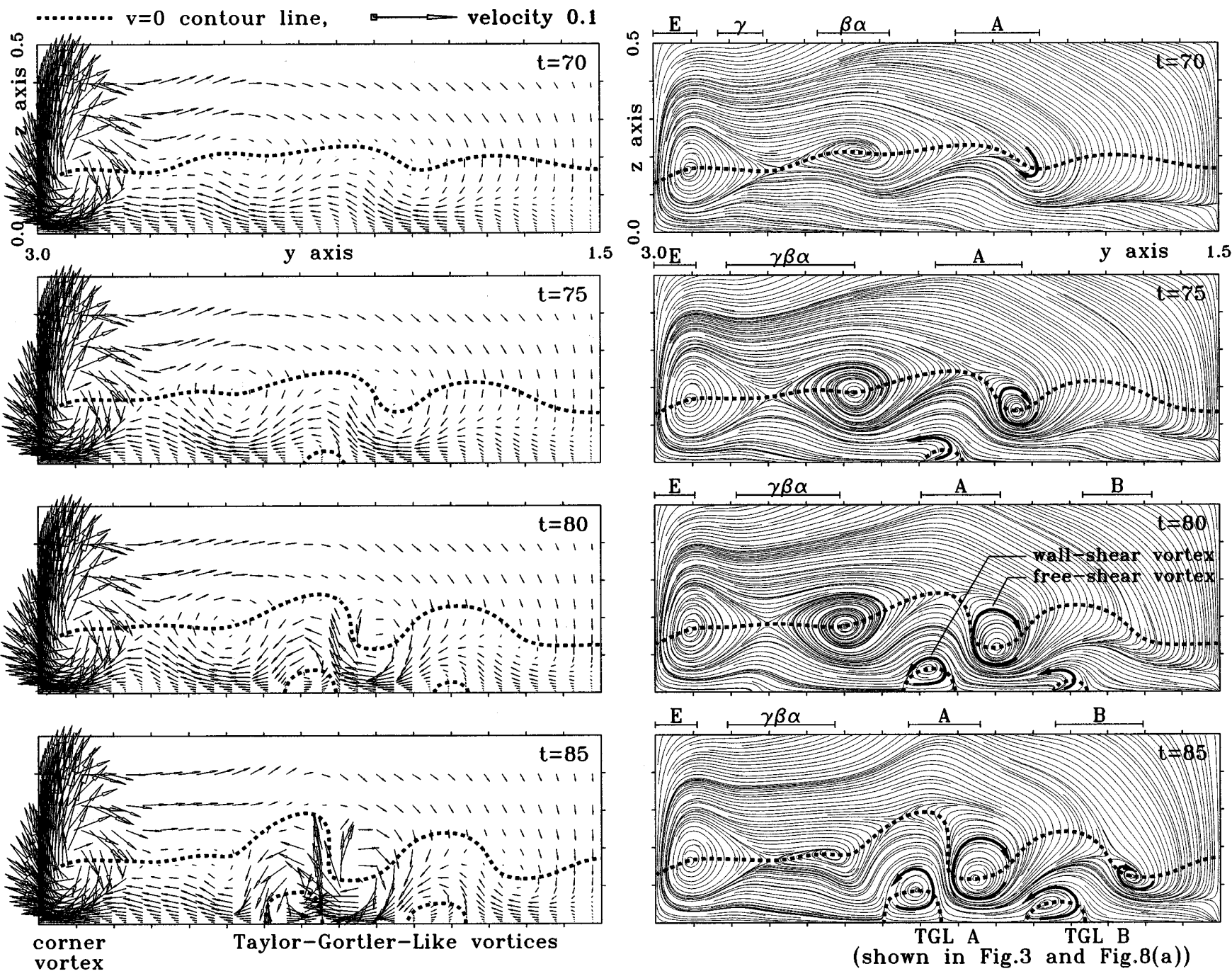

a Velocity vector plots

(shown in Fig.3 and Fig.8(a))

b Streamline plots

Fig. 20a,b. The formation of TGL vortices at the $x=0.525$ plane $(70 \leq t \leq 85)$. a velocity vector plots; $\mathbf{b}$ streamline plots

surface, and the separation surface are closely related. The spatial location where the USE has marked variation in size is consistent with the most disrupted $v=0$ contour surface. It is exactly at this spatial location where the separation surface detaches from the upstream side wall. In the vicinity of the distorted $v=0$ contour surface, the sign-switching spanwise velocity induces a free-shear vortex. The pressure field established to support the existence of this vortex further affects the boundary layer of the outward-running spiraling flow in the sense that a wall-shear vortex is formed near the floor of the cavity. This pair of well-established vortices, referred to as Taylor-Görtler vortices, bursts from the spatial location which has the local maximum kinetic energy.

\section{References}

Abdallah, S. (1987): Numerical solution for the incompressible Navier-Stokes equations in primitive variables using a non-staggered grid, II. J. Comput. Phys. 70, 193-202

Aidun, C. K.; Triantafillopoulos, N. G.; Benson, J. D. (1991):

Global stability of a lid-driven cavity with throughflow: Flow visualization studies. Phys. Fluids A3, 2081-2091
Arnal, M.; Lauer, O.; Lilek, Z.; Peric, M. (1992): Prediction of three-dimensional lid driven cavity flow. Deville, M. et al. (eds.) A GAMM-Workshop, NNFM, 36, 13-24

Babu, V.; Korpela, S. A. (1994): Numerical solutions of the incompressible, three-dimensional Navier-Stokes equations. Comput. Fluids 23 (5), 675-691

Burggraf, O. R. (1966): Analytical and numerical studies of the structure of steady separated flows. J. Fluid Mech. 24 (1), 113-151 Chiang, T. P.; Sheu, W. H.; Hwang, R. R. (1997): Three-dimensional vortex dynamics in a shear-driven rectangular cavity. Int. J. Comput. Fluid Dyn. 8, 201-214

Chiang, T. P.; Hwang, R. R.; Sheu, W. H. (1996): On end-wall corner vortices in a lid-driven cavity. ASME, J. Fluids Eng. 119, 201-204

Cortes, A. B.; Miller, J. D. (1994): Numerical experiments with the lid-driven cavity flow problem. Compu. Fluids 23 (8), 1005-1027 Deng, G. B.; Piquet, J.; Queutey, P.; Visonneau, M. (1994): A new fully coupled solution of the Navier-Stokes equations. Int. J. Num. Meth. Fluids 19, 605-639

Deville, M.; Lê, T.-H.; Morchoisne, Y. (eds.) (1992): Numerical Simulation of 3-D Incompressible Unsteady Viscous Laminar Flows. Notes Num. Fluids Mech. 36, A GAMM-Workshop

Ethier, C. R.; Steinman, D. A. (1994): Exact fully 3D Navier-Stokes solutions for benchmarking. Int. J. Num. Meth. Fluids 19, 369-375 
Freitas, C. J.; Street, R. L. (1988): Non-linear transport phenomena in a complex recirculating flow: A numerical investigation. Int. J. Num. Meth. Fluids 8, 769-802

Freitas, C. J.; Street, R. L.; Findikakis, A. N.; Koseff, J. R. (1985): Numerical simulation of three-dimensional flow in a cavity. Int. J. Num. Meth. Fluids 5, 561-575

Görtler, H. (1940): Über eine dreidimensionale Insta laminarer Grenzschichten an konkaven Wänden. Nachr. Akad. Wiss. Göttingen, Math. Phys. Kl. Neue Folge I, 2, 1-26

Kato, Y.; Kawai, H.; Tanahashi, T. (1990): Numerical flow analysis in a cubic cavity by the GSMAC finite element method. JSME Int. J. Series II, 33, No. 4, 649-658

Koseff, J. R.; Street, R. L. (1982): Visualization studies of a shear driven three-dimensional recirculating flow. Three dimensional Turbulent Shear Driven Flow, ASME: 23-31

Koseff, J. R.; Street, R. L. (1984a): Visualization studies of a shear driven three-dimensional recirculating flow. ASME J. Fluids Eng. $106,21-29$

Koseff, J. R.; Street, R. L. (1984b): On end wall effects in a liddriven cavity flow. ASME J. Fluids Eng. 106, 385-389

Koseff, J. R.; Street, R. L. (1984c): The lid-driven cavity flow: A synthesis of qualitative and quantitative observations. ASME J. Fluids Eng. 106, 390-398

Koseff, J. R.; Street, R. L.; Gresho, P. M.; Upson, C. D.; Humphrey, J. A. C.; To, W. M. (1983): A three-dimensional lid-driven cavity flow: experiment and simulation. Proc. 3rd Int. Conf. Num. Meth. Lam. and Turb. Flow, Seattle, August: 564-581

Kost, A.; Mitra, N. K.; Fiebig, M.; Bochum, R. U. (1992): Nu-

merical simulation of three-dimensional unsteady flow in a cavity. Deville, M. et al. (eds.) A GAMM-Workshop, NNFM 36, 79-90
Ku, H. C.; Hirsh, R. S.; Taylor, T. D. (1987): A pseudospectral method for solution of the three-dimensional incompressible Navier-Stokes equations, J. Comput. Phys. 70, 439-462

Ladyzhenskaya, O. A. (1963): Mathematical Problems in the Dynamics of a Viscous Incompressible Flow. Gordon \& Breach, New York

Leonard, B. P. (1979): A stable and accurate convective modeling procedure based on quadratic upstream interpolation. Comput. Meth. Appl. Mech. Eng. 19, 59-98

Patankar, S. V. (1980): Numerical Heat Transfer and Fluid Flow. Hemisphere

Perng, C. Y.; Street, R. L. (1989): Three-dimensional unsteady flow simulations: alternative strategies for a volume-averaged calculation. Int. J. Num. Meth. Fluids 9, 341-362

Prasad, A. K.; Koseff, J. R. (1989): Reynolds number and endwall effects on a lid-driven cavity flow. Phys. Fluids A1, 208218

Prasad, A. K.; Perng, C. Y.; Koseff, J. Y. (1988): Some observations of the influence of longitudinal vortices in a lid-driven cavity flow. Proc. First Nat. Fluid Dynamics Congr. Cincinnati, Ohio: 288-295

Rhee, H. S.; Koseff, J. R.; Street, R. L. (1984): Flow visualization of a recirculating flow by rheoscopic liquid and liquid crystal techniques. Exp. Fluids, 2, 57-64

Sheu, W. H.; Lee, S. M. (1996): A segregated solution algorithm for incompressible flows in general co-ordinates. Int. J. Num. Meth. Fluids. 22, 515-548

Zang, Y.; Street, R. L.; Koseff, J. R. (1993): A dynamic mixed subgridscale model and its application to turbulent recirculating flows. Phys. Fluids A5 (12), 3186-3196 